\title{
Data report: bulk mineralogical composition of Cascadia margin sediments, IODP Expedition $311^{1}$
}

\author{
André Bahr, ${ }^{2}$ Gerhard Bohrmann, ${ }^{2}$ and Barbara M.A. Teichert ${ }^{3}$
}

\section{Chapter contents}

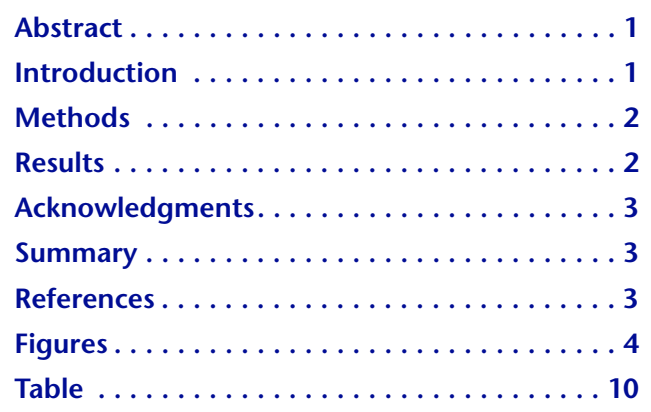

${ }^{1}$ Bahr, A., Bohrmann, G., and Teichert, B.M.A., 2008. Data report: bulk mineralogical composition of Cascadia margin sediments, IODP Expedition 311. In Riedel, M., Collett, T.S., Malone, M.J., and the Expedition 311 Scientists, Proc. IODP, 311: Washington, DC (Integrated Ocean Drilling Program Management International, Inc.). doi:10.2204/iodp.proc.311.201.2008 ${ }^{2}$ Forschungszentrum Ozeanränder, Universität Bremen, Postfach 330440, D-28334 Bremen, Germany. bahr@uni-bremen.de ${ }^{3}$ Bundesanstalt für Geowissenschaften und Rohstoffe, Stilleweg 2, D-30655 Hannover, Germany.

\begin{abstract}
The bulk mineralogical compositions of sediment samples from Sites U1325-U1329, northern Cascadia margin, were determined by X-ray diffraction analyses. The results document a highly variable mineralogy dominated by detrital components, mainly quartz, feldspar, mica, and clay minerals. Carbonate minerals are present only in minor amounts $(<5 \mathrm{wt} \%)$. The long-term variations in the relative abundance of each mineral correspond to the particular lithostratigraphic units.
\end{abstract}

\section{Introduction}

The main scope of Integrated Ocean Drilling Program Expedition 311 was to evaluate gas hydrate formation models in subduction zone accretionary prisms ("Expedition 311 summary" chapter, this volume). For this purpose a transect of four sites (from southwest to northeast, Sites U1326, U1325, U1327, and U1329) was cored through the northern Cascadia margin off Vancouver Island, representing different stages in the evolution of gas hydrate formation in an accretionary prism from its most recent stage at the deformation front in the southwest (Site U1326) to its final stage in shallower waters in the northeast (Site U1329). Additionally, a cold vent (Site U1328) with active fluid flow was investigated.

Corresponding to the tectonic setting, a vertical and lateral highly variable lithology was cored, with frequent intercalation of turbiditic sediments and extensive active faulting near the deformation front (Davis and Hyndman, 1989). Both turbidites and faulting are important for the gas hydrate distribution in the sediment column, whereas faults or fractures act as a fluid conduit; as proposed especially for Site U1328 ("Expedition 311 summary" chapter, this volume; Riedel et al., 2006), coarse-grained turbidites may provide the pore space needed for gas hydrate accumulation. Qualitative assessment of the bulk mineralogy could therefore give additional information about gas hydrate formation processes and might be useful for the study of authigenic carbonates that formed associated with gas hydrates (e.g., Bohrmann et al., 1998). 


\section{Methods}

A total of 309 sediment samples from Sites U1325-U1329 were dried and crushed in an agate mortar. After adding a 1:5 ratio of corundum as an internal standard, the sample was homogenized with acetone and prepared as randomly oriented powder slides. X-ray diffraction (XRD) analyses were performed using a Philips PW 1710 instrument $\left(\mathrm{CoK}_{\alpha}\right)$ at the Alfred Wegener Institute for Polar and Marine Research in Bremerhaven (Germany) with $40 \mathrm{kV}$ accelerator voltage and $40 \mathrm{~mA}$ current. Scans were run from $3^{\circ}$ to $100^{\circ} 2 \theta$ at a scanning speed of $0.02^{\circ} / \mathrm{s}$. The interpretation was done using the MacDiff 4.2.2 program by Rainer Petschik (servermac.geologie.unifrankfurt.de/Rainer.html). The calcite content (in weight percent) was estimated using calibration curves for the calcite/corundum ratio. The $\mathrm{MgCO}_{3}$ content of calcite (in molar percent) was determined using the shift in the d(104) peak (Lumsden, 1979). The results for the mineral abundances are given in a qualitative way (modified after Vogt, 1997) as peak intensity $(I)$ or integrated peak area $(A)$ relative to $I$ or $A$ of the corundum standard (reflection at $2.085 \AA$ [113]).

\section{Results}

XRD analyses show that the sediments from Sites U1325-U1329 are dominated by detrital minerals, mainly quartz, feldspar (plagioclase and potassium feldspar combined), mica, and clay minerals (Table T1). Carbonates are only minor components constituting $<5 \mathrm{wt} \%$, with low $\mathrm{Mg}$ calcite as the dominant phase. Dolomite is generally rare, and aragonite is absent at the detection limit. Because of the inhomogeneous nature of the sediment (Expedition 311 Scientists, 2005) the mineralogical composition varies considerably between adjacent sampling depths and also between adjacent holes of one site (cf. Site U1329). Nevertheless, the overall mineralogical trends reflect the lithostratigraphic division applied for each site, as described in the following (in southwest-northeast succession).

At Site U1326, situated at the southwest end of the transect, low calcite content discriminates lithostratigraphic Unit II from Units I and III, which contain more calcite (Fig. F1). This conforms with low microfossil contents in Unit II compared to those in Unit I and III. Grain size variations are less prominent than at Site U1325 (see the "Site U1326" chapter, this volume); thus, changes in the mineralogy are less distinct, although a trend toward higher illite and chlorite contents in the lower Unit II and entire Unit III can be observed.
Site U1325 is situated in a slope basin near the southwest end of the transect cored during Expedition 311 and comprises four lithostratigraphic units (see the "Site U1325" chapter, this volume). As expected from the basinal setting, turbidites are frequent throughout the cored sections. Unit I is divided into Subunit IA, consisting of sand-rich deposits with abundant quartz and feldspar, and Subunit IB, dominated by fine-grained sediments with high illite and chlorite contents. Grain size and mineralogy are apparently closely coupled in both cases (Fig. F2). Unit II is distinguished from Subunit IB by the absence of diatoms, sponge spicules, and foraminifers as well as low calcite and illite contents. In Unit III, where biogenic components are more abundant, calcite increases again and finally drops to very low values in Unit IV. The maximum amounts of calcite found in Subunit IB and Unit III correspond to high amounts of biogenic components, including calcareous microfossils such as foraminifers. The calcite content may therefore originate mainly from calcareous microfossil remains.

At Site U1327, located midslope of the accretionary prism, the most prominent variations are recorded in the illite content (Fig. F3). Lithostratigraphic Unit I (clay and silty clay interbedded with coarse silt to gravel-sized layers) has fluctuating low to high illite contents, which are more constant on a relatively low level in Unit II (clay to silty clay with diatoms and interbedded sandy silt and sand layers) and increase again in Unit III (silty clay with diatoms in the lower part). The calcite content is low throughout the record. In both Units II and III dolomite was observed regularly, although in minor amounts.

Site U1328 is located at the Bullseye vent site, within an area of active cold vents. The bulk of the emanating methane gas is thought to be transported along isolated feeder channels or fractures toward the seafloor (Riedel et al., 2006), leading to a massive accumulation of gas hydrate in the upper $\sim 40 \mathrm{~m}$ of the sedimentary column (see the "Site U1328" chapter, this volume). The mineralogy of this zone is marked by a relatively high quartz abundance and a zone of very high chlorite and illite contents between 25 and 35 meters below seafloor (mbsf) (Fig. F4); small amounts of dolomite are present occasionally. Although steeply dipping structural features are common in the upper $40 \mathrm{~m}$ of the sedimentary column, the mineralogical variations between Holes U1328B, U1328C, U1328D, and U1328E are relatively small (Fig. F4). The mineralogy of Site U1328 below 40 mbsf is quite variable because of the abundance of turbiditic deposits. Lithostratigraphic Unit I (turbidite and sand rich) and Unit II (fine-grained, microfossil-rich sediments) are mainly differentiated by 
higher illite and calcite ratios in Unit II relative to Unit I. Quartz contents do not follow the lithologic units very closely but show a more cyclic pattern with contents that are high at $\sim 30 \mathrm{mbsf}$, decrease until $\sim 100 \mathrm{msbf}$, increase again in the lower Unit I and upper Unit II, and decrease to a second low at the Unit II/III boundary. Quartz remains on a moderate level in Unit III after an initial increase. The mineralogical composition of samples from Unit III (abyssal plain deposits with very few turbidites) shows a lower variability than the turbidite-rich Units I and II. Notable are relatively high calcite contents and the frequent occurrence of minor amounts of dolomite. The calcite content at Site U1328 might partly reflect biogenic components but possibly also dispersed authigenic precipitated calcite crystals, especially near the bottom-simulating reflector and in the gas hydrate accumulation zone in the upper 40 mbsf.

From Site U1329, located at the eastern limit of the gas hydrate occurrence on the northern Cascadia margin, active dolomite formation is reported (see the "Site U1329" chapter, this volume); thus, the high dolomite contents (in relation to the lower part of the cores) observed in Unit I (Fig. F5) might be caused by authigenic dolomite precipitation. Also clearly visible is the hiatus between upper Miocene (Unit III) and Pleistocene (Unit II) deposits, accompanied by a drop in feldspar, chlorite, calcite, dolomite, and less pronounced, illite contents. The low frequency of turbidite deposits in Unit III is reflected by a rather constant mineralogical composition.

Crossplots of the corundum-normalized intensities of calcite versus quartz and chlorite versus illite (Fig. F6) show that all sites share a similar lithology; no site-specific clusters or trends are displayed. An exception is Site U1325 with generally higher quartz contents than the other sites, which is explained by the high abundance of coarse-grained turbidites. Although the abundances of illite and chlorite are positively correlated, suggesting a common detrital origin, quartz and calcite intensities are uncorrelated, likely because the calcite is a mixture of detrital, biogenic, and authigenic origins.

\section{Summary}

The mineralogical composition of sediment samples from Sites U1325-U1329 is dominated by detrital minerals such as quartz, feldspar, and clay minerals, with quartz and feldspar dominating lithologic sections rich in turbidites. The calcite content, which is generally very low, increases within lithologic units containing high amounts of calcareous microfossils but never exceeds $5 \mathrm{wt} \%$. Elevated calcite contents observed in some sections of the active venting Site U1328 correspond to the observed occurrence of authigenic carbonates. The data therefore represent primarily the detrital background sedimentation at the specific sites.

\section{Acknowledgments}

The authors thank Rita Fröhlking and Susanne Wiebe (both Alfred Wegener Institute, Bremerhaven) for help with the XRD analyses and Thierry Adatte, Mitch Malone, and editor Lorri Peters for their careful review of the manuscript. This research used samples and/or data provided by the Integrated Ocean Drilling Program (IODP). This research was funded by grant BO 1049/8 of the Deutsche Forschungsgemeinschaft. This is RCOM publication number 0540 .

\section{References}

Bohrmann, G., Greinert, J., Suess, E., and Torres, M., 1998. Authigenic carbonates from the Cascadia subduction zone and their relation to gas hydrate stability, Geology, 26(7):647-650. doi:10.1130/0091-

7613(1998)026<0647:ACFTCS $>2.3$. CO;2

Davis, E.E., and Hyndman, R.D., 1989. Accretion and recent deformation of sediments along the northern Cascadia subduction zone. Geol. Soc. Am. Bull., 101(11):1465-1480. doi:10.1130/00167606(1989)101<1465:AARDOS>2.3.CO;2

Expedition 311 Scientists, 2005. Cascadia margin gas hydrates. IODP Prel. Rept., 311. doi:10.2204/ iodp.pr.311.2005

Lumsden, D.S., 1979. Discrepancy between thin section and X-ray estimates of dolomite in limestone. J. Sediment. Petrol., 49:429-436.

Riedel, M., Novosel, I., Spence, G.D., Hyndman, R.D., Chapman, R.N., Solem, R.C., and Lewis, T., 2006. Geophysical and geochemical signatures associated with gas hydrate-related venting in the northern Cascadia margin. Geol. Soc. Am. Bull., 118(1):23-38. doi:10.1130/ B25720.1

Vogt, C., 1997. Zeitliche und räumliche Verteilung von Mineralvergesellschaftungen in spätquartären Sedimenten des Arktischen Ozeans und ihre Nützlichkeit als Klimaindikatoren während der Glazial/InterglazialWechsel. Berichte zur Polarforschung, Vol. 251, Bremerhaven, Germany (Alfred-Wegener-Institut für Polarund Meeresforschung).

Initial receipt: 31 May 2007

Acceptance: 23 October 2007

Publication: 5 February 2008

MS 311-201 
Figure F1. Downhole variations of X-ray diffraction peak intensity $(I)$ or peak area $(A)$ of quartz (Qz), feldspar (Fsp), chlorite (Chl), illite (Ill), calcite (Cc), and dolomite (Dol) relative to the standard corundum (Cor) for Holes U1326C and U1326D. BSR = bottom-simulating reflector.

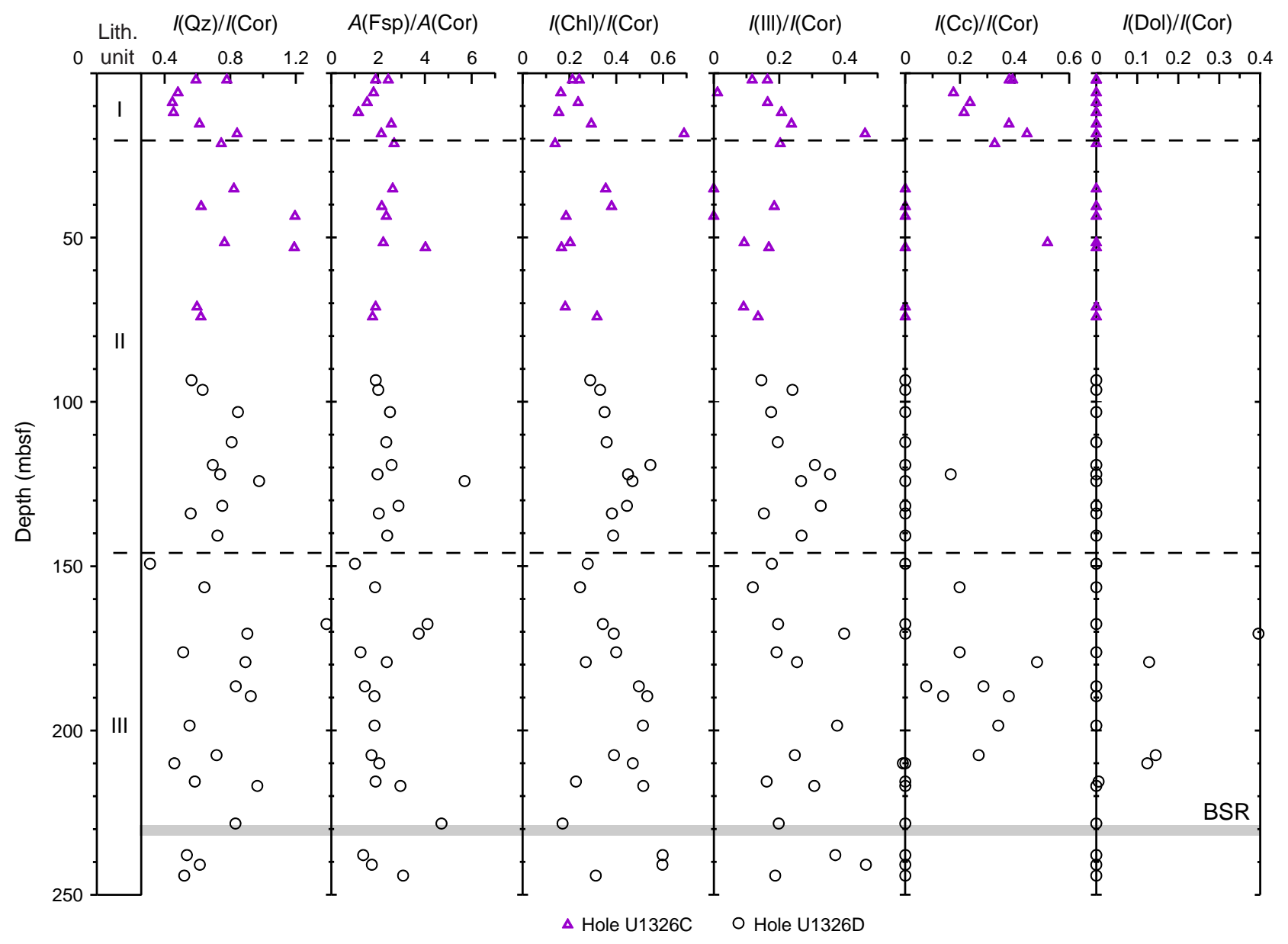


Figure F2. Downhole variations of X-ray diffraction peak intensity $(I)$ or peak area $(A)$ of quartz (Qz), feldspar (Fsp), chlorite (Chl), illite (IIl), calcite (Cc), and dolomite (Dol) relative to the standard corundum (Cor) for Holes U1325B, U1325C, and U1325D. BSR = bottom-simulating reflector.

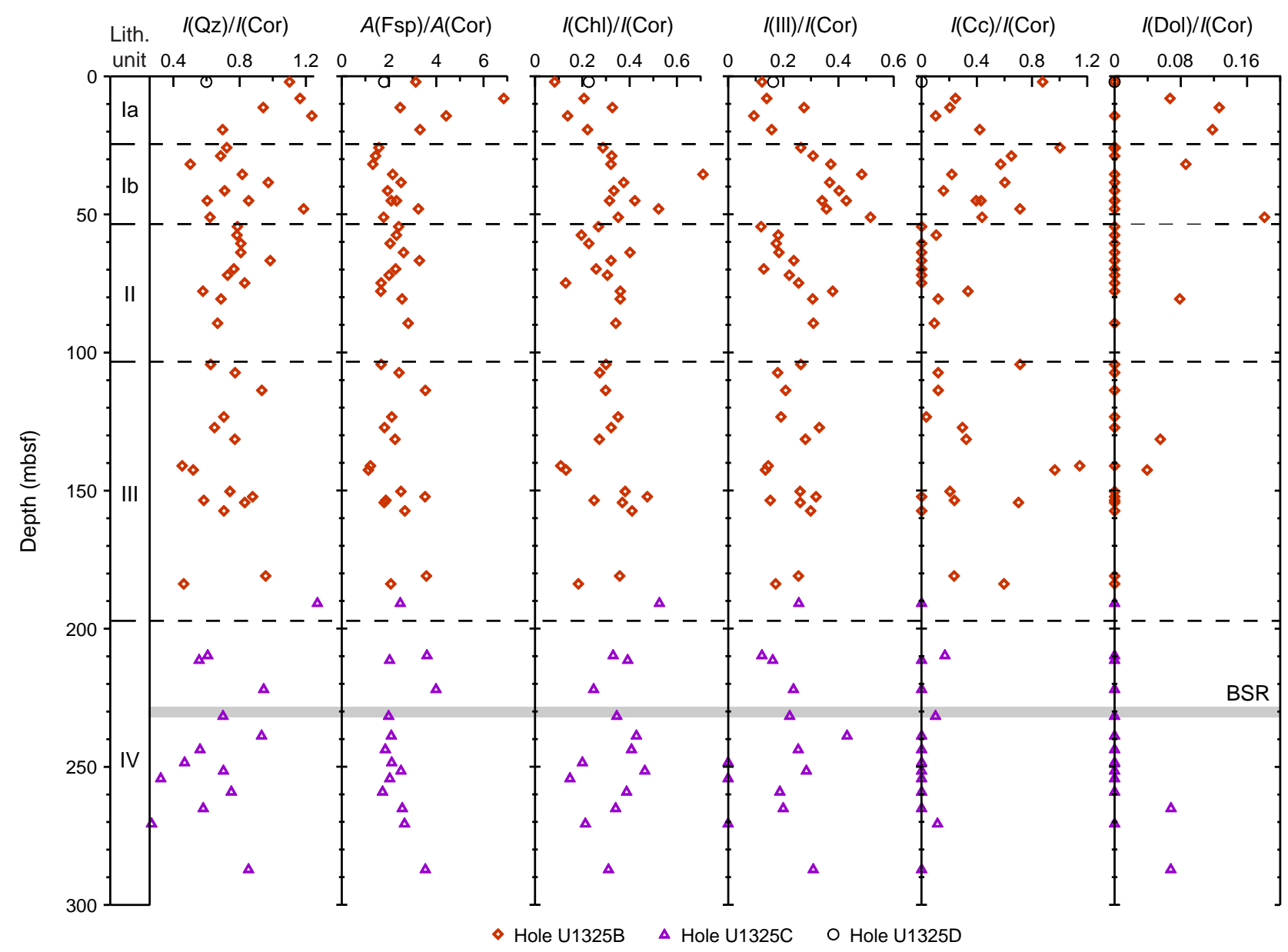


Figure F3. Downhole variations of X-ray diffraction peak intensity $(I)$ or peak area $(A)$ of quartz (Qz), feldspar (Fsp), chlorite (Chl), illite (Ill), calcite (Cc), and dolomite (Dol) relative to the standard corundum (Cor) for Hole U1327C. BSR = bottom-simulating reflector.

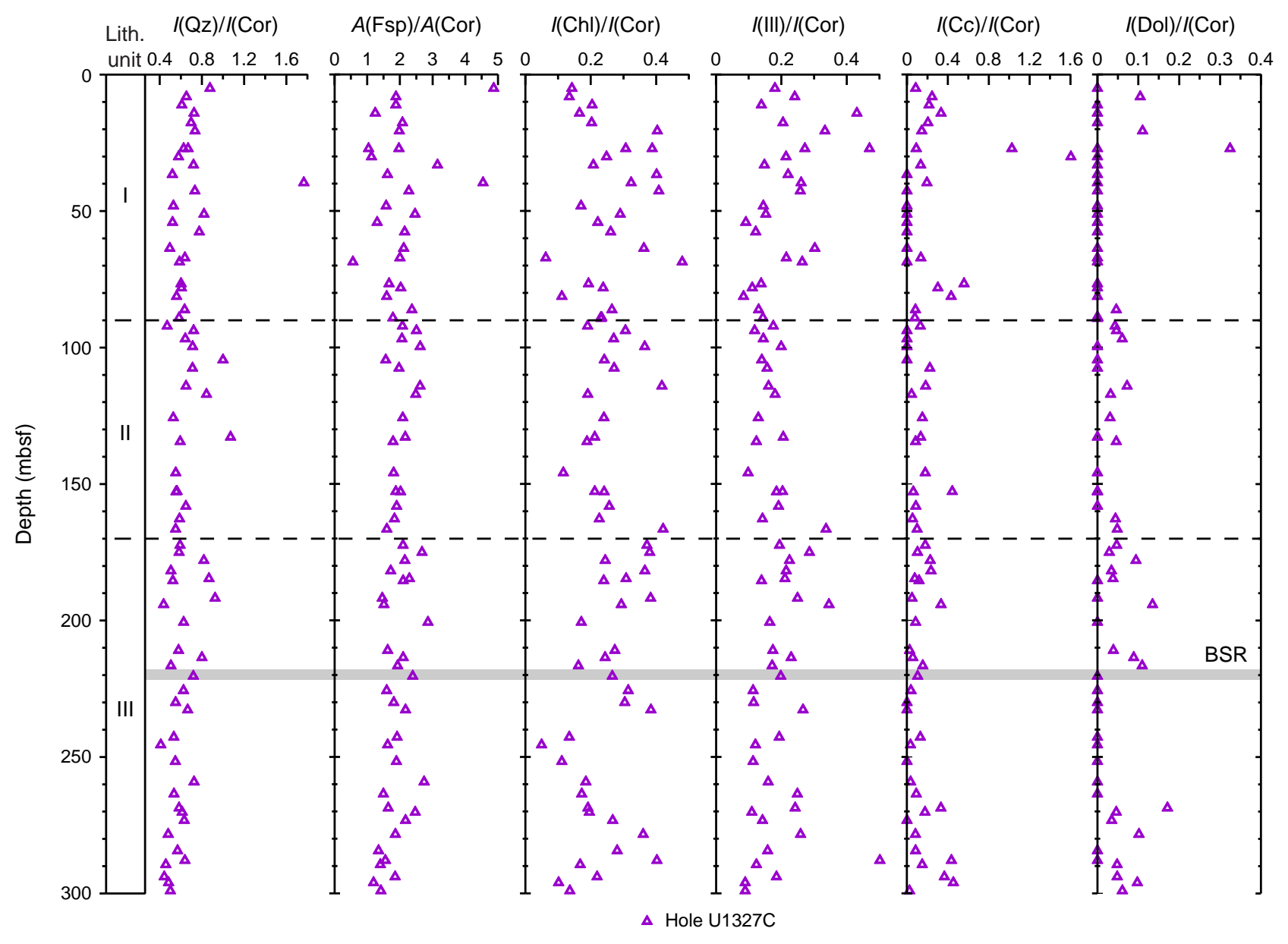


Figure F4. Downhole variations of X-ray diffraction peak intensity $(I)$ or peak area $(A)$ of quartz (Qz), feldspar (Fsp), chlorite (Chl), illite (Ill), calcite (Cc), and dolomite (Dol) relative to the standard corundum (Cor) for Holes U1328B, U1328C, U1328D, and U1328E. BSR = bottom-simulating reflector.

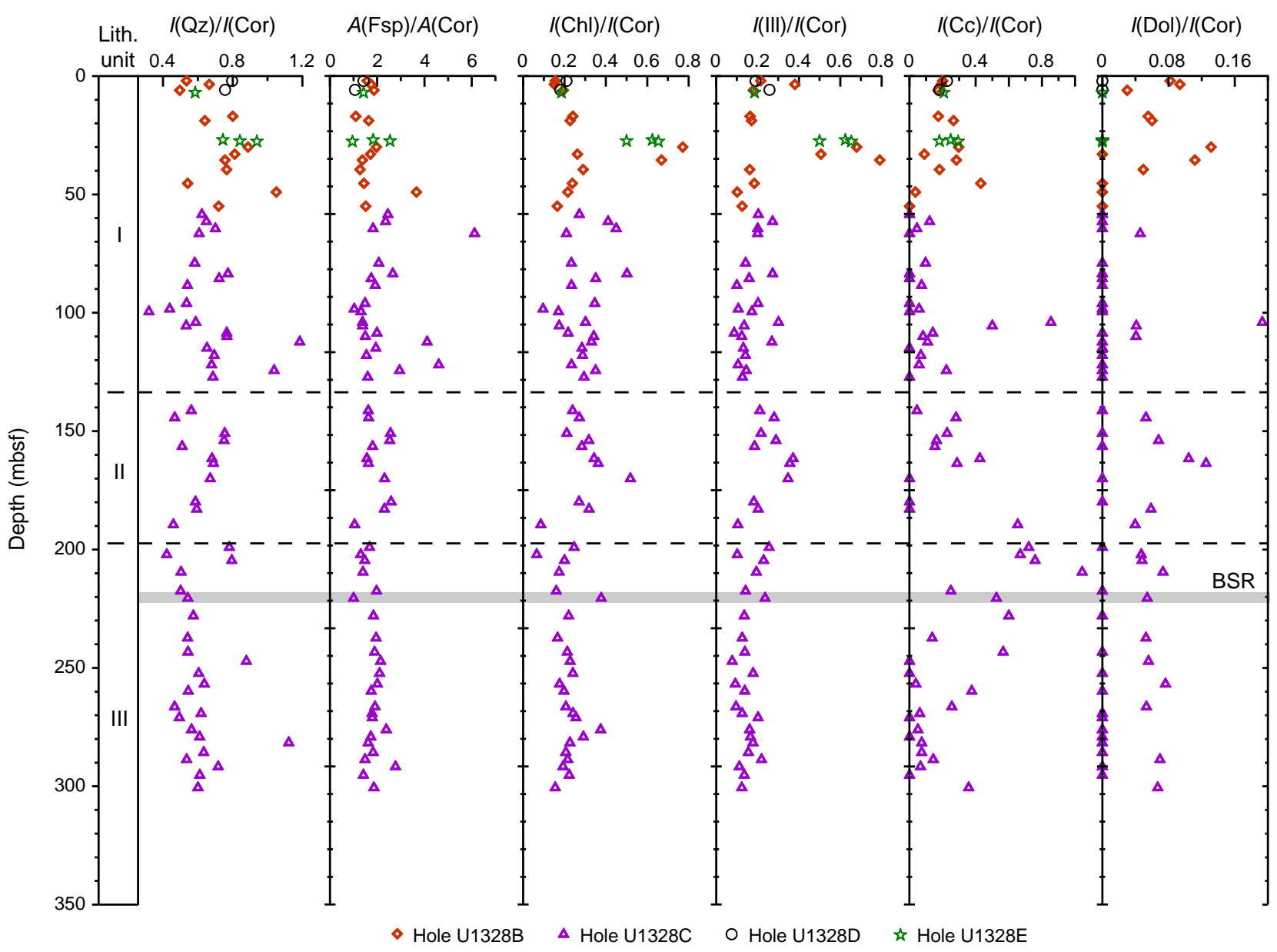


Figure F5. Downhole variations of X-ray diffraction peak intensity $(I)$ or peak area $(A)$ of quartz (Qz), feldspar (Fsp), chlorite (Chl), illite (Ill), calcite (Cc), and dolomite (Dol) relative to the standard corundum (Cor) for Holes U1329B, U1329C, and U1329E. BSR = bottom-simulating reflector.

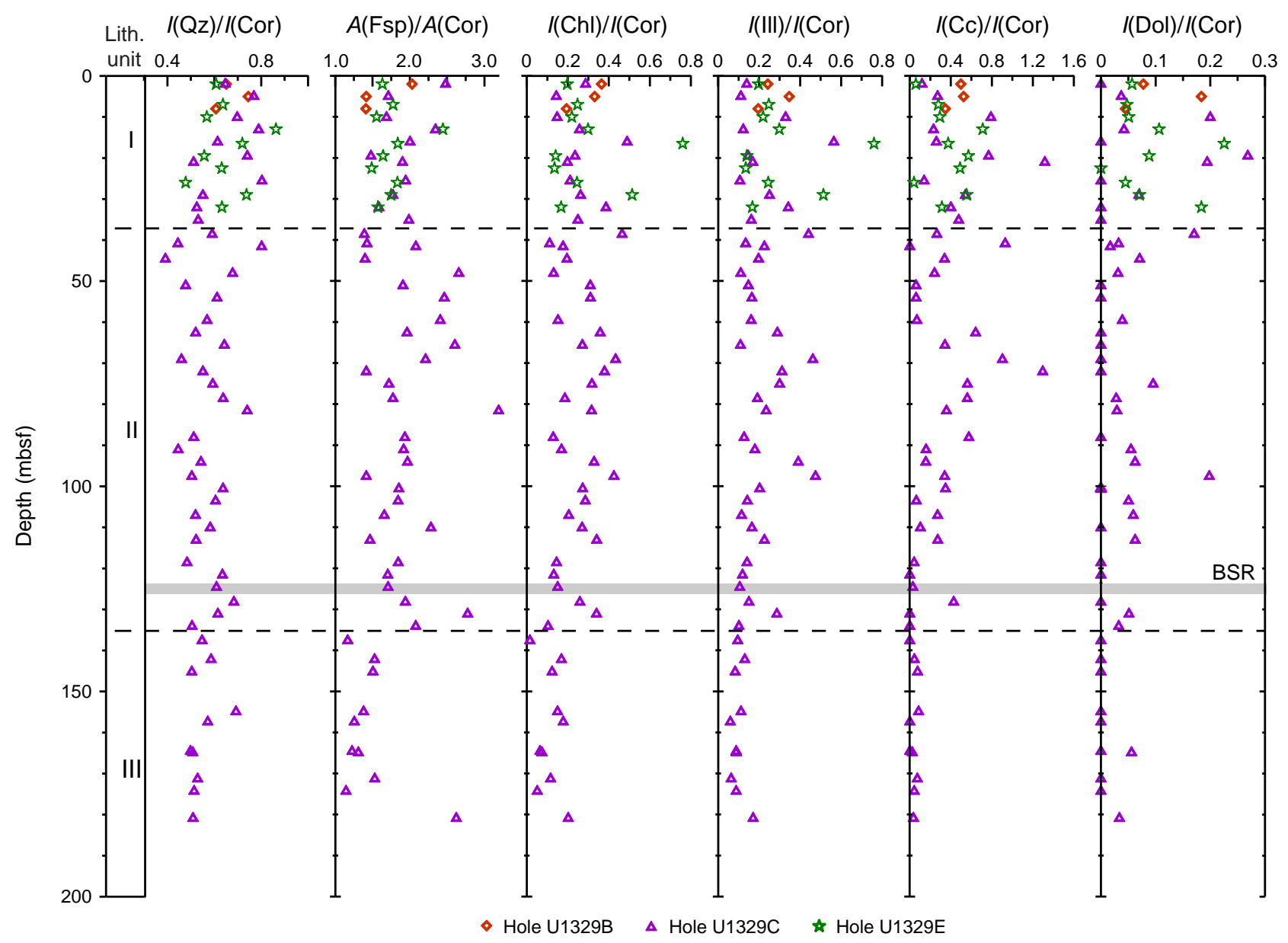


Figure F6. Cross-plots of the intensities of (A) calcite versus quartz and (B) chlorite versus illite, each relative to the (113) corundum peak.
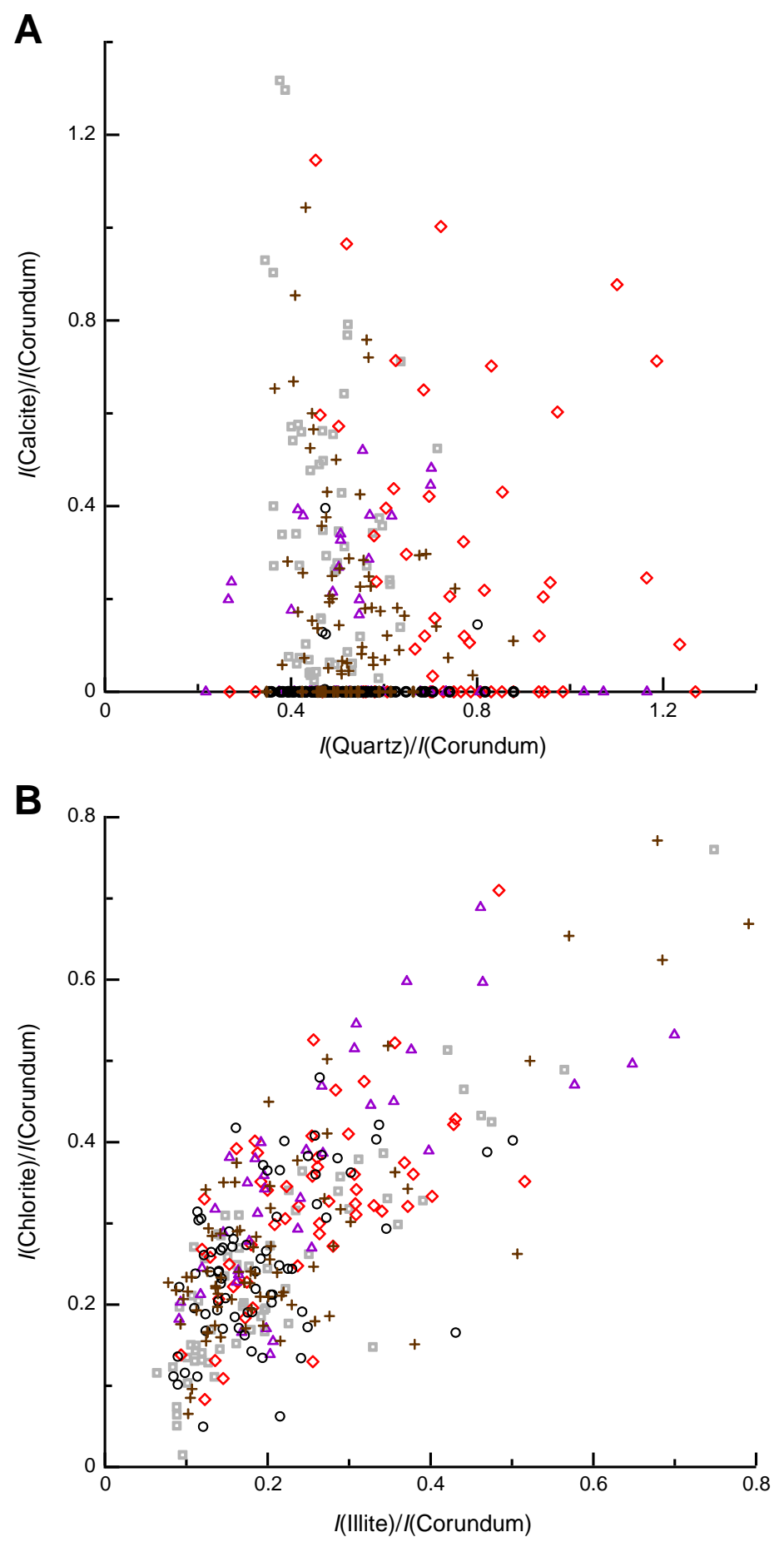

$\diamond$ Hole U1325 $\Delta$ Hole U1326 $\odot$ Hole U1327 + Hole U1328 $\square$ Hole U1329 
Table T1. Mineral composition of bulk sediment samples. (See table note. Continued on next four pages.)

\begin{tabular}{|c|c|c|c|c|c|c|c|c|c|c|c|}
\hline \multirow{2}{*}{$\begin{array}{l}\text { Core, section, } \\
\text { interval }(\mathrm{cm})\end{array}$} & \multirow{2}{*}{$\begin{array}{l}\text { Depth } \\
\text { (mbsf) }\end{array}$} & \multirow{2}{*}{$\begin{array}{l}\text { l, quartz } \\
(4.26 \AA)\end{array}$} & \multirow{2}{*}{$\begin{array}{c}\text { A, feldspars } \\
(3.18-3.24 \AA)\end{array}$} & \multirow{2}{*}{$\begin{array}{l}\text { I, chlorite } \\
(3.54 \AA)\end{array}$} & \multirow{2}{*}{$\begin{array}{l}\text { l, illite } \\
(10 \AA)\end{array}$} & \multirow{2}{*}{$\begin{array}{c}\text { l, pyrite } \\
(2.706 \AA)\end{array}$} & \multirow{2}{*}{$\begin{array}{l}\text { I, calcite } \\
\mathrm{d}(104)\end{array}$} & \multirow{2}{*}{$\begin{array}{l}\text { I, dolomite } \\
\mathrm{d}(104)\end{array}$} & & Calci & \\
\hline & & & & & & & & & (mol\%) & $\mathrm{d}(104)(\AA ̊)$ & (wt\%) \\
\hline 311-U1325B- & & & & & & & & & & & \\
\hline $1 \mathrm{H}-2,49-51$ & 1.99 & 1.10 & 3.13 & 0.08 & 0.12 & 0.11 & 0.88 & 0.00 & 0.83 & 3.0335 & 1.9 \\
\hline $1 \mathrm{H}-6,50-52$ & 8 & 1.16 & 6.85 & 0.21 & 0.14 & 0.05 & 0.25 & 0.07 & & & 0.6 \\
\hline $2 \mathrm{H}-2,50-52$ & 11.3 & 0.94 & 2.47 & 0.33 & 0.28 & 0.10 & 0.20 & 0.13 & & & 0.5 \\
\hline $2 \mathrm{H}-4,50-52$ & 14.3 & 1.24 & 4.42 & 0.14 & 0.09 & 0.20 & 0.10 & 0.00 & & & 0.2 \\
\hline $3 X-1,50-52$ & 19.3 & 0.70 & 3.31 & 0.22 & 0.16 & 0.00 & 0.42 & 0.12 & 0.83 & 3.0335 & 0.9 \\
\hline $4 \mathrm{H}-2,50-52$ & 25.82 & 0.72 & 1.58 & 0.29 & 0.26 & 0.17 & 1.00 & 0.00 & 1.97 & 3.0301 & 1.9 \\
\hline $4 \mathrm{H}-4,50-52$ & 28.82 & 0.69 & 1.43 & 0.32 & 0.31 & 0.07 & 0.65 & 0.00 & 1.97 & 3.0301 & 1.2 \\
\hline $4 \mathrm{H}-6,50-52$ & 31.82 & 0.50 & 1.32 & 0.32 & 0.37 & 0.13 & 0.57 & 0.09 & 0.83 & 3.0335 & 1.1 \\
\hline $5 \mathrm{H}-2,50-52$ & 35.5 & 0.82 & 2.16 & 0.71 & 0.48 & 0.09 & 0.22 & 0.00 & & & 0.4 \\
\hline $5 \mathrm{H}-4,50-52$ & 38.42 & 0.97 & 2.51 & 0.37 & 0.37 & 0.27 & 0.60 & 0.00 & 5.37 & 3.0199 & 1.2 \\
\hline $5 \mathrm{H}-6,50-52$ & 41.42 & 0.71 & 1.94 & 0.33 & 0.40 & 0.11 & 0.16 & 0.00 & & & 0.3 \\
\hline $6 \mathrm{H}-2,52-54$ & 45.02 & 0.60 & 2.10 & 0.31 & 0.34 & 0.07 & 0.40 & 0.00 & 0.83 & 3.0335 & 0.8 \\
\hline $6 \mathrm{H}-4,50-52$ & 48 & 1.19 & 3.24 & 0.52 & 0.36 & 0.26 & 0.71 & 0.00 & & & 0.8 \\
\hline $6 \mathrm{H}-6,50-52$ & 51 & 0.62 & 1.77 & 0.35 & 0.52 & 0.07 & 0.44 & 0.18 & 1.40 & 3.0318 & 0.9 \\
\hline $7 \mathrm{H}-2,41-43$ & 54.41 & 0.79 & 2.41 & 0.27 & 0.12 & 0.14 & 0.00 & 0.00 & & & 0.0 \\
\hline $7 \mathrm{H}-4,50-52$ & 57.5 & 0.78 & 2.32 & 0.20 & 0.18 & 0.09 & 0.11 & 0.00 & & & 0.2 \\
\hline $7 \mathrm{H}-6,50-52$ & 60.5 & 0.81 & 2.05 & 0.23 & 0.17 & 0.13 & 0.00 & 0.00 & & & 0.0 \\
\hline $8 \mathrm{H}-2,50-52$ & 63.76 & 0.81 & 2.62 & 0.40 & 0.18 & 0.10 & 0.00 & 0.00 & & & 0.0 \\
\hline $8 \mathrm{H}-4,46-48$ & 66.72 & 0.98 & 3.29 & 0.32 & 0.24 & 0.11 & 0.00 & 0.00 & & & 0.0 \\
\hline $8 \mathrm{H}-6,46-48$ & 69.72 & 0.77 & 2.28 & 0.26 & 0.13 & 0.12 & 0.00 & 0.00 & & & 0.0 \\
\hline $9 \mathrm{X}-1,50-52$ & 72 & 0.73 & 2.00 & 0.31 & 0.22 & 0.08 & 0.00 & 0.00 & & & 0.0 \\
\hline $10 X-2,50-52$ & 74.8 & 0.83 & 1.67 & 0.13 & 0.26 & 0.05 & 0.00 & 0.00 & & & 0.0 \\
\hline $10 \times-4,50-52$ & 77.8 & 0.58 & 1.65 & 0.36 & 0.38 & 0.11 & 0.34 & 0.00 & & & 0.7 \\
\hline $10 X-6,50-52$ & 80.6 & 0.69 & 2.55 & 0.36 & 0.31 & 0.09 & 0.12 & 0.08 & & & 0.3 \\
\hline $12 X-4,50-52$ & 89.4 & 0.67 & 2.81 & 0.34 & 0.31 & 0.11 & 0.09 & 0.00 & & & 0.2 \\
\hline $14 X-2,50-52$ & 104.3 & 0.63 & 1.67 & 0.30 & 0.26 & 0.09 & 0.71 & 0.00 & 0.83 & 3.0335 & 1.4 \\
\hline $14 X-4,50-52$ & 107.3 & 0.77 & 2.43 & 0.27 & 0.18 & 0.17 & 0.12 & 0.00 & & & 0.3 \\
\hline $15 \times-2,50-52$ & 113.7 & 0.93 & 3.54 & 0.30 & 0.21 & 0.09 & 0.12 & 0.00 & & & 0.3 \\
\hline $16 X-2,50-52$ & 123.3 & 0.70 & 2.12 & 0.35 & 0.19 & 0.21 & 0.03 & 0.00 & & & 0.1 \\
\hline $16 \times-6,50-52$ & 127.15 & 0.65 & 1.81 & 0.32 & 0.33 & 0.12 & 0.30 & 0.00 & & & 0.6 \\
\hline $18 X-1,50-52$ & 131.4 & 0.77 & 2.26 & 0.27 & 0.28 & 0.20 & 0.32 & 0.06 & & & 0.7 \\
\hline $19 \times-1,50-52$ & 141.01 & 0.45 & 1.22 & 0.11 & 0.15 & 0.10 & 1.15 & 0.00 & 1.40 & 3.0318 & 2.4 \\
\hline $19 X-2,50-52$ & 142.51 & 0.52 & 1.13 & 0.13 & 0.14 & 0.12 & 0.97 & 0.04 & 1.97 & 3.0301 & 1.9 \\
\hline $20 X-1,8-10$ & 150.28 & 0.74 & 2.51 & 0.38 & 0.26 & 0.12 & 0.21 & 0.00 & & & 0.4 \\
\hline $20 X-2,50-52$ & 152.2 & 0.88 & 3.53 & 0.47 & 0.32 & 0.14 & 0.00 & 0.00 & & & 0.0 \\
\hline $20 X-3,32-34$ & 153.52 & 0.58 & 1.87 & 0.25 & 0.15 & 0.19 & 0.24 & 0.00 & & & 0.6 \\
\hline $20 X-4,46-48$ & 154.3 & 0.83 & 1.80 & 0.37 & 0.26 & 0.00 & 0.70 & 0.00 & 1.43 & 3.0317 & 1.5 \\
\hline $20 X-6,48-50$ & 157.32 & 0.71 & 2.67 & 0.41 & 0.30 & 0.08 & 0.00 & 0.00 & & & 0.0 \\
\hline $24 X-2,50-52$ & 180.89 & 0.96 & 3.58 & 0.36 & 0.25 & 0.00 & 0.24 & 0.00 & & & 0.5 \\
\hline $24 X-4,50-52$ & 183.77 & 0.46 & 2.08 & 0.18 & 0.17 & 0.00 & 0.60 & 0.00 & 1.43 & 3.0317 & 1.6 \\
\hline 311-U1325C- & & & & & & & & & & & \\
\hline $1 X-2,50-52$ & 190.8 & 1.27 & 2.48 & 0.53 & 0.26 & 0.00 & 0.00 & 0.00 & & & 0.0 \\
\hline $4 X-2,50-52$ & 209.74 & 0.61 & 3.61 & 0.33 & 0.12 & 0.19 & 0.00 & 0.00 & & & 0.4 \\
\hline $4 X-4,50-52$ & 211.42 & 0.56 & 2.03 & 0.39 & 0.16 & 0.18 & 0.00 & 0.00 & & & 0.0 \\
\hline $6 X-4,50-52$ & 222.01 & 0.95 & 3.99 & 0.25 & 0.24 & 0.16 & 0.00 & 0.00 & & & 0.0 \\
\hline $7 X-4,50-52$ & 231.7 & 0.70 & 1.98 & 0.35 & 0.22 & 0.10 & 0.00 & 0.00 & & & 0.0 \\
\hline $8 X-2,50-52$ & 238.8 & 0.93 & 2.10 & 0.43 & 0.43 & 0.11 & 0.00 & 0.00 & & & 0.0 \\
\hline $8 X-6,50-52$ & 243.8 & 0.56 & 1.85 & 0.41 & 0.25 & 0.11 & 0.00 & 0.00 & & & 0.0 \\
\hline $9 X-2,50-52$ & 248.5 & 0.47 & 2.12 & 0.20 & 0.00 & 0.28 & 0.00 & 0.00 & & & 0.0 \\
\hline $9 X-4,50-52$ & 251.5 & 0.70 & 2.51 & 0.46 & 0.28 & 0.00 & 0.00 & 0.00 & & & 0.0 \\
\hline $9 X-6,50-52$ & 254.28 & 0.32 & 2.04 & 0.15 & 0.00 & 0.26 & 0.00 & 0.00 & & & 0.0 \\
\hline $11 X-2,50-52$ & 259.1 & 0.75 & 1.73 & 0.39 & 0.19 & 0.00 & 0.00 & 0.00 & & & 0.0 \\
\hline $11 X-6,50-52$ & 265.1 & 0.58 & 2.56 & 0.34 & 0.20 & 0.11 & 0.00 & 0.07 & & & 0.0 \\
\hline $12 X-4,50-52$ & 270.67 & 0.27 & 2.66 & 0.21 & 0.00 & 0.37 & 0.00 & 0.00 & & & 0.0 \\
\hline $14 X-3,50-52$ & 287.2 & 0.85 & 3.54 & 0.31 & 0.31 & 0.14 & 0.00 & 0.07 & & & 0.0 \\
\hline 311-U1325D- & & & & & & & & & & & \\
\hline $1 X-2,50-52$ & 2 & 0.60 & 1.79 & 0.23 & 0.16 & 0.14 & 0.00 & 0.00 & & & 0.0 \\
\hline 311-U1326C- & & & & & & & & & & & \\
\hline $1 \mathrm{H}-2,49-51$ & 1.99 & 0.78 & 1.90 & 0.21 & 0.12 & 0.24 & 0.38 & 0.00 & 0.83 & 3.0335 & 0.8 \\
\hline $1 \mathrm{H}-2,50-52$ & 2 & 0.59 & 2.43 & 0.24 & 0.16 & 0.00 & 0.39 & 0.00 & 0.27 & 3.0352 & 1.0 \\
\hline $2 \mathrm{H}-2,46-48$ & 5.9 & 0.48 & 1.81 & 0.16 & 0.01 & 0.14 & 0.18 & 0.00 & & & 0.5 \\
\hline $2 \mathrm{H}-4,46-48$ & 8.86 & 0.45 & 1.53 & 0.24 & 0.16 & 0.24 & 0.24 & 0.00 & & & 0.7 \\
\hline $2 \mathrm{H}-6,50-52$ & 11.9 & 0.45 & 1.16 & 0.15 & 0.21 & 0.10 & 0.22 & 0.00 & & & 0.4 \\
\hline $3 \mathrm{H}-2,50-52$ & 15.4 & 0.61 & 2.56 & 0.29 & 0.24 & 0.00 & 0.38 & 0.00 & 0.83 & 3.0335 & 0.8 \\
\hline $3 \mathrm{H}-4,50-52$ & 18.4 & 0.84 & 2.14 & 0.69 & 0.46 & 0.00 & 0.45 & 0.00 & 2.10 & 3.0297 & 1.0 \\
\hline $3 \mathrm{H}-6,50-52$ & 21.4 & 0.75 & 2.68 & 0.14 & 0.20 & 0.00 & 0.33 & 0.00 & & & 0.7 \\
\hline
\end{tabular}


Table T1 (continued).

\begin{tabular}{|c|c|c|c|c|c|c|c|c|c|c|c|}
\hline \multirow{2}{*}{$\begin{array}{l}\text { Core, section, } \\
\text { interval }(\mathrm{cm})\end{array}$} & \multirow{2}{*}{$\begin{array}{l}\text { Depth } \\
\text { (mbsf) }\end{array}$} & \multirow{2}{*}{$\begin{array}{l}\text { l, quartz } \\
(4.26 \AA)\end{array}$} & \multirow{2}{*}{$\begin{array}{c}\text { A, feldspars } \\
(3.18-3.24 \AA)\end{array}$} & \multirow{2}{*}{$\begin{array}{c}\text { I, chlorite } \\
(3.54 \AA)\end{array}$} & \multirow{2}{*}{$\begin{array}{l}\text { l, illite } \\
(10 \AA)\end{array}$} & \multirow{2}{*}{$\begin{array}{c}\text { l, pyrite } \\
(2.706 \AA)\end{array}$} & \multirow{2}{*}{$\begin{array}{l}\text { I, calcite } \\
\text { d(104) }\end{array}$} & & $\mathrm{MqCO}_{3}$ & Calcit & \\
\hline & & & & & & & & $\mathrm{d}(104)$ & $(\mathrm{mol} \%)$ & $\mathrm{d}(104)(\AA)$ & (wt\%) \\
\hline $5 X-4,50-52$ & 35.15 & 0.82 & 2.63 & 0.35 & 0.00 & 0.20 & 0.00 & 0.00 & & & 0.0 \\
\hline $6 X-1,50-52$ & 40.5 & 0.62 & 2.16 & 0.38 & 0.18 & 0.15 & 0.00 & 0.00 & & & 0.0 \\
\hline $6 X-3,50-52$ & 43.5 & 1.20 & 2.35 & 0.19 & 0.00 & 0.31 & 0.00 & 0.00 & & & 0.0 \\
\hline $7 X-2,50-52$ & 51.53 & 0.76 & 2.22 & 0.20 & 0.09 & 0.12 & 0.52 & 0.00 & 1.43 & 3.0317 & 1.1 \\
\hline $7 X-3,50-52$ & 53.01 & 1.19 & 4.02 & 0.17 & 0.17 & 0.00 & 0.00 & 0.00 & & & 0.0 \\
\hline $9 X-2,50-52$ & 71.1 & 0.60 & 1.90 & 0.18 & 0.09 & 0.00 & 0.00 & 0.00 & & & 0.0 \\
\hline $9 X-4,50-52$ & 74.1 & 0.62 & 1.77 & 0.32 & 0.14 & 0.00 & 0.00 & 0.00 & & & 0.0 \\
\hline 311-U1326D- & & & & & & & & & & & \\
\hline $2 X-4,50-52$ & 93.4 & 0.56 & 1.90 & 0.29 & 0.15 & 0.07 & 0.00 & 0.00 & & & 0.0 \\
\hline $2 X-6,50-52$ & 96.34 & 0.63 & 2.01 & 0.33 & 0.24 & 0.11 & 0.00 & 0.00 & & & 0.0 \\
\hline $3 X-4,50-52$ & 103.1 & 0.85 & 2.51 & 0.35 & 0.18 & 0.00 & 0.00 & 0.00 & & & 0.0 \\
\hline $4 X-4,50-52$ & 112.26 & 0.81 & 2.35 & 0.36 & 0.20 & 0.00 & 0.00 & 0.00 & & & 0.0 \\
\hline $5 X-2,50-52$ & 119.2 & 0.69 & 2.58 & 0.55 & 0.31 & 0.00 & 0.00 & 0.00 & & & 0.0 \\
\hline $5 X-4,50-52$ & 122.04 & 0.74 & 1.98 & 0.45 & 0.35 & 0.19 & 0.17 & 0.00 & & & 0.3 \\
\hline $5 X-6,50-52$ & 124.1 & 0.98 & 5.69 & 0.47 & 0.27 & 0.13 & 0.00 & 0.00 & & & 0.0 \\
\hline $6 X-4,50-52$ & 131.63 & 0.75 & 2.87 & 0.45 & 0.33 & 0.17 & 0.00 & 0.00 & & & 0.0 \\
\hline $6 X-6,50-52$ & 133.95 & 0.56 & 2.03 & 0.38 & 0.15 & 0.19 & 0.00 & 0.00 & & & 0.0 \\
\hline $7 X-4,50-52$ & 140.69 & 0.72 & 2.39 & 0.39 & 0.27 & 0.13 & 0.00 & 0.00 & & & 0.0 \\
\hline $8 X-3,50-52$ & 149.26 & 0.31 & 1.01 & 0.28 & 0.18 & 0.12 & 0.00 & 0.00 & & & 0.0 \\
\hline $9 X-1,50-52$ & 156.4 & 0.64 & 1.87 & 0.25 & 0.12 & 0.10 & 0.20 & 0.00 & & & 0.4 \\
\hline $10 X-2,50-52$ & 167.6 & 1.39 & 4.11 & 0.34 & 0.20 & 0.05 & 0.00 & 0.00 & & & 0.0 \\
\hline $10 X-4,50-52$ & 170.52 & 0.90 & 3.74 & 0.39 & 0.40 & 0.00 & 0.00 & 0.40 & & & 0.0 \\
\hline $11 X-2,50-52$ & 176.19 & 0.51 & 1.25 & 0.40 & 0.19 & 0.00 & 0.20 & 0.00 & & & 0.5 \\
\hline $11 X-4,50-52$ & 179.19 & 0.89 & 2.37 & 0.27 & 0.25 & 0.00 & 0.48 & 0.13 & 0.27 & 3.0352 & 1.1 \\
\hline $12 X-2,50-52$ & 186.55 & 0.83 & 1.44 & 0.50 & 0.65 & 0.00 & 0.29 & 0.00 & & & 0.5 \\
\hline $12 X-4,50-52$ & 189.55 & 0.93 & 1.85 & 0.53 & 0.70 & 0.09 & 0.38 & 0.00 & 1.97 & 3.0301 & 0.8 \\
\hline $13 X-4,50-52$ & 198.5 & 0.55 & 1.85 & 0.51 & 0.38 & 0.13 & 0.34 & 0.00 & & & 0.7 \\
\hline $14 X-4,50-52$ & 207.5 & 0.72 & 1.72 & 0.39 & 0.25 & 0.00 & 0.27 & 0.14 & & & 0.6 \\
\hline $14 X-6,50-52$ & 209.98 & 0.46 & 2.05 & 0.47 & 0.58 & 0.16 & 0.00 & 0.12 & & & 0.0 \\
\hline $15 X-2,50-52$ & 215.52 & 0.58 & 1.89 & 0.23 & 0.16 & 0.12 & 0.00 & 0.01 & & & 0.0 \\
\hline $15 X-3,50-52$ & 216.86 & 0.97 & 2.96 & 0.52 & 0.31 & 0.45 & 0.00 & 0.00 & & & 0.0 \\
\hline $16 X-4,50-52$ & 228.3 & 0.83 & 4.71 & 0.17 & 0.20 & 0.00 & 0.00 & 0.00 & & & 0.0 \\
\hline $17 X-4,50-52$ & 237.9 & 0.54 & 1.37 & 0.60 & 0.37 & 0.00 & 0.00 & 0.00 & & & 0.0 \\
\hline $17 X-6,50-52$ & 240.84 & 0.61 & 1.74 & 0.60 & 0.46 & 0.00 & 0.00 & 0.00 & & & 0.0 \\
\hline $18 X-2,50-52$ & 244.15 & 0.52 & 3.07 & 0.31 & 0.19 & 0.17 & 0.00 & 0.00 & & & 0.0 \\
\hline 311-U1327C- & & & & & & & & & & & \\
\hline $1 \mathrm{H}-4,50-52$ & 5 & 0.88 & 4.87 & 0.14 & 0.18 & 0.00 & 0.08 & 0.00 & & & 0.2 \\
\hline $2 \mathrm{H}-2,50-52$ & 8.1 & 0.65 & 1.88 & 0.13 & 0.24 & 0.10 & 0.25 & 0.10 & & & 0.5 \\
\hline $2 \mathrm{H}-4,50-52$ & 11.1 & 0.61 & 1.88 & 0.20 & 0.14 & 0.08 & 0.22 & 0.00 & & & 0.4 \\
\hline $2 \mathrm{H}-6,50-52$ & 14.1 & 0.73 & 1.25 & 0.17 & 0.43 & 0.00 & 0.33 & 0.00 & & & 0.6 \\
\hline $3 \mathrm{H}-2,50-52$ & 17.6 & 0.70 & 2.08 & 0.20 & 0.20 & 0.15 & 0.20 & 0.00 & & & 0.4 \\
\hline $3 \mathrm{H}-4,50-52$ & 20.6 & 0.73 & 1.98 & 0.40 & 0.33 & 0.12 & 0.14 & 0.11 & & & 0.3 \\
\hline $3 \mathrm{H}-6,50-52$ & 27.1 & 0.67 & 1.97 & 0.39 & 0.47 & 0.17 & 0.09 & 0.00 & & & 0.2 \\
\hline $4 \mathrm{H}-2,50-52$ & 27.1 & 0.63 & 1.04 & 0.31 & 0.27 & 0.11 & 1.03 & 0.32 & 0.27 & 3.0352 & 2.1 \\
\hline $4 \mathrm{H}-4,50-52$ & 30.1 & 0.58 & 1.13 & 0.25 & 0.21 & 0.10 & 1.60 & 0.00 & 0.83 & 3.0335 & 3.1 \\
\hline $4 \mathrm{H}-6,50-52$ & 33.1 & 0.72 & 3.15 & 0.21 & 0.15 & 0.14 & 0.13 & 0.00 & & & 0.3 \\
\hline $5 \mathrm{H}-2,50-52$ & 36.6 & 0.52 & 1.62 & 0.40 & 0.22 & 0.18 & 0.00 & 0.00 & & & 0.0 \\
\hline $5 \mathrm{H}-4,50-52$ & 39.6 & 1.77 & 4.54 & 0.32 & 0.26 & 0.00 & 0.19 & 0.00 & & & 0.4 \\
\hline $5 \mathrm{H}-6,49-51$ & 42.59 & 0.73 & 2.27 & 0.41 & 0.26 & 0.13 & 0.00 & 0.00 & & & 0.0 \\
\hline $7 \mathrm{H}-2,50-52$ & 48.1 & 0.53 & 1.58 & 0.17 & 0.14 & 0.00 & 0.00 & 0.00 & & & 0.0 \\
\hline $7 \mathrm{H}-4,50-52$ & 51.1 & 0.82 & 2.46 & 0.29 & 0.15 & 0.15 & 0.00 & 0.00 & & & 0.0 \\
\hline $7 \mathrm{H}-6,50-52$ & 54.1 & 0.52 & 1.31 & 0.22 & 0.09 & 0.08 & 0.00 & 0.00 & & & 0.0 \\
\hline $8 \mathrm{H}-2,50-52$ & 57.6 & 0.77 & 2.14 & 0.26 & 0.12 & 0.09 & 0.00 & 0.00 & & & 0.0 \\
\hline $8 \mathrm{H}-6,50-52$ & 63.6 & 0.50 & 2.11 & 0.36 & 0.30 & 0.00 & 0.00 & 0.00 & & & 0.0 \\
\hline $9 \mathrm{H}-2,50-52$ & 67.1 & 0.64 & 2.00 & 0.06 & 0.22 & 0.00 & 0.14 & 0.00 & & & 0.3 \\
\hline $9 \mathrm{H}-3,50-52$ & 68.6 & 0.59 & 0.56 & 0.48 & 0.26 & 0.19 & 0.00 & 0.00 & & & 0.0 \\
\hline $10 \mathrm{H}-2,49-51$ & 76.59 & 0.60 & 1.67 & 0.19 & 0.14 & 0.15 & 0.56 & 0.00 & 2.27 & 3.0292 & 1.1 \\
\hline $10 \mathrm{H}-3,49-51$ & 78.09 & 0.61 & 2.03 & 0.24 & 0.11 & 0.15 & 0.30 & 0.00 & & & 0.6 \\
\hline $10 \mathrm{H}-6,49-51$ & 81.24 & 0.56 & 1.60 & 0.11 & 0.08 & 0.16 & 0.43 & 0.00 & 1.40 & 3.0318 & 1.0 \\
\hline $11 \mathrm{H}-2,50-52$ & 86.1 & 0.64 & 2.37 & 0.26 & 0.13 & 0.32 & 0.08 & 0.05 & & & 0.2 \\
\hline $11 \mathrm{H}-4,50-52$ & 89.1 & 0.58 & 1.79 & 0.23 & 0.14 & 0.10 & 0.08 & 0.00 & & & 0.2 \\
\hline $11 \mathrm{H}-6,50-52$ & 92.1 & 0.47 & 2.08 & 0.19 & 0.18 & 0.13 & 0.13 & 0.04 & & & 0.3 \\
\hline $12 X-2,50-52$ & 93.76 & 0.72 & 2.50 & 0.31 & 0.12 & 0.10 & 0.00 & 0.05 & & & 0.0 \\
\hline $12 X-4,50-52$ & 96.76 & 0.64 & 2.06 & 0.27 & 0.14 & 0.09 & 0.00 & 0.06 & & & 0.0 \\
\hline $12 X-6,50-52$ & 99.62 & 0.71 & 2.62 & 0.37 & 0.20 & 0.14 & 0.00 & 0.00 & & & 0.0 \\
\hline $13 X-2,50-52$ & 104.5 & 1.00 & 1.57 & 0.24 & 0.14 & 0.11 & 0.00 & 0.00 & & & 0.0 \\
\hline $13 X-4,50-52$ & 107.5 & 0.71 & 1.97 & 0.27 & 0.16 & 0.29 & 0.22 & 0.00 & & & 0.5 \\
\hline $14 X-2,50-52$ & 114.1 & 0.65 & 2.61 & 0.42 & 0.16 & 0.08 & 0.18 & 0.07 & & & 0.4 \\
\hline
\end{tabular}


Table T1 (continued).

\begin{tabular}{|c|c|c|c|c|c|c|c|c|c|c|c|}
\hline \multirow{2}{*}{$\begin{array}{l}\text { Core, section, } \\
\text { interval }(\mathrm{cm})\end{array}$} & \multirow{2}{*}{$\begin{array}{l}\text { Depth } \\
\text { (mbsf) }\end{array}$} & \multirow{2}{*}{$\begin{array}{l}\text { I, quartz } \\
(4.26 \AA)\end{array}$} & \multirow{2}{*}{$\begin{array}{c}\text { A, feldspars } \\
(3.18-3.24 \AA)\end{array}$} & \multirow{2}{*}{$\begin{array}{c}\text { I, chlorite } \\
(3.54 \AA)\end{array}$} & \multirow{2}{*}{$\begin{array}{l}\text { I, illite } \\
(10 \AA)\end{array}$} & \multirow{2}{*}{$\begin{array}{c}\text { l, pyrite } \\
(2.706 \AA)\end{array}$} & \multirow{2}{*}{$\begin{array}{l}\text { I, calcite } \\
\text { d(104) }\end{array}$} & & $\mathrm{MgCO}_{3}$ & Calci & \\
\hline & & & & & & & & $d(104)$ & $(\mathrm{mol} \%)$ & $\mathrm{d}(104)(\AA)$ & (wt\%) \\
\hline $14 X-4,50-52$ & 117.1 & 0.84 & 2.49 & 0.19 & 0.18 & 0.12 & 0.04 & 0.03 & & & 0.1 \\
\hline $16 X-2,50-52$ & 125.7 & 0.53 & 2.09 & 0.24 & 0.13 & 0.14 & 0.15 & 0.03 & & & 0.3 \\
\hline $17 X-2,46-48$ & 132.77 & 1.07 & 2.17 & 0.21 & 0.21 & 0.11 & 0.13 & 0.00 & & & 0.3 \\
\hline $17 X-4,50-52$ & 134.42 & 0.59 & 1.79 & 0.19 & 0.12 & 0.09 & 0.08 & 0.05 & & & 0.2 \\
\hline $18 X-4,50-52$ & 145.85 & 0.55 & 1.81 & 0.12 & 0.10 & 0.10 & 0.18 & 0.00 & & & 0.4 \\
\hline $19 X-2,42-44$ & 152.73 & 0.56 & 1.87 & 0.21 & 0.20 & 0.13 & 0.44 & 0.00 & 0.83 & 3.0335 & 0.9 \\
\hline $19 X-2,50-52$ & 152.81 & 0.55 & 2.02 & 0.24 & 0.18 & 0.09 & 0.06 & 0.00 & & & 0.1 \\
\hline $19 X-6,50-52$ & 158.1 & 0.65 & 1.90 & 0.26 & 0.19 & 0.08 & 0.09 & 0.00 & & & 0.2 \\
\hline $20 X-2,41-43$ & 162.71 & 0.59 & 1.83 & 0.23 & 0.14 & 0.09 & 0.05 & 0.04 & & & 0.1 \\
\hline $20 X-5,41-43$ & 166.49 & 0.55 & 1.60 & 0.42 & 0.34 & 0.10 & 0.10 & 0.05 & & & 0.2 \\
\hline $21 X-2,50-52$ & 172.4 & 0.59 & 2.09 & 0.37 & 0.19 & 0.10 & 0.18 & 0.05 & & & 0.4 \\
\hline $21 X-4,47-49$ & 174.99 & 0.58 & 2.67 & 0.38 & 0.29 & 0.13 & 0.10 & 0.03 & & & 0.2 \\
\hline $21 X-6,52-54$ & 177.94 & 0.82 & 2.15 & 0.24 & 0.22 & 0.10 & 0.23 & 0.09 & & & 0.5 \\
\hline $22 X-2,50-52$ & 181.77 & 0.51 & 1.72 & 0.37 & 0.21 & 0.08 & 0.23 & 0.03 & & & 0.5 \\
\hline $22 X-4,50-52$ & 184.6 & 0.87 & 2.29 & 0.31 & 0.21 & 0.21 & 0.08 & 0.04 & & & 0.2 \\
\hline $22 X-5,50-52$ & 185.37 & 0.52 & 2.10 & 0.24 & 0.14 & 0.12 & 0.12 & 0.00 & & & 0.2 \\
\hline $23 X-2,46-48$ & 191.77 & 0.92 & 1.46 & 0.38 & 0.25 & 0.07 & 0.05 & 0.00 & & & 0.1 \\
\hline $23 X-4,43-45$ & 194.13 & 0.44 & 1.51 & 0.29 & 0.35 & 0.16 & 0.33 & 0.13 & & & 0.7 \\
\hline $25 X-2,50-52$ & 200.61 & 0.63 & 2.86 & 0.17 & 0.16 & 0.23 & 0.08 & 0.00 & & & 0.2 \\
\hline $26 X-2,50-52$ & 210.9 & 0.58 & 1.63 & 0.27 & 0.17 & 0.19 & 0.03 & 0.04 & & & 0.1 \\
\hline $26 X-4,50-52$ & 213.54 & 0.80 & 2.10 & 0.24 & 0.23 & 0.12 & 0.06 & 0.09 & & & 0.1 \\
\hline $26 X-6,50-52$ & 216.54 & 0.51 & 1.93 & 0.16 & 0.17 & 0.06 & 0.15 & 0.11 & & & 0.4 \\
\hline $27 X-2,50-52$ & 220.38 & 0.72 & 2.39 & 0.27 & 0.20 & 0.12 & 0.10 & 0.00 & & & 0.2 \\
\hline $27 X-6,50-52$ & 225.63 & 0.62 & 1.59 & 0.31 & 0.11 & 0.09 & 0.04 & 0.00 & & & 0.1 \\
\hline $28 X-2,40-42$ & 230 & 0.55 & 1.81 & 0.30 & 0.11 & 0.12 & 0.00 & 0.00 & & & 0.0 \\
\hline $28 X-4,50-52$ & 232.7 & 0.67 & 2.17 & 0.38 & 0.27 & 0.14 & 0.00 & 0.00 & & & 0.0 \\
\hline $29 X-4,50-52$ & 242.7 & 0.53 & 1.91 & 0.13 & 0.19 & 0.07 & 0.13 & 0.00 & & & 0.3 \\
\hline $29 X-6,48-50$ & 245.53 & 0.41 & 1.63 & 0.05 & 0.12 & 0.08 & 0.03 & 0.00 & & & 0.1 \\
\hline $30 \times-4,50-52$ & 251.61 & 0.55 & 1.89 & 0.11 & 0.11 & 0.09 & 0.00 & 0.00 & & & 0.0 \\
\hline $31 X-2,50-52$ & 259.1 & 0.73 & 2.74 & 0.18 & 0.16 & 0.23 & 0.04 & 0.00 & & & 0.1 \\
\hline $31 X-5,46-48$ & 263.52 & 0.54 & 1.49 & 0.17 & 0.25 & 0.14 & 0.09 & 0.00 & & & 0.2 \\
\hline $32 X-2,45-47$ & 268.65 & 0.58 & 1.64 & 0.19 & 0.24 & 0.05 & 0.33 & 0.17 & 1.70 & 3.0309 & 0.8 \\
\hline $32 X-2,50-52$ & 270.2 & 0.61 & 2.47 & 0.20 & 0.11 & 0.13 & 0.18 & 0.05 & & & 0.4 \\
\hline $32 X-5,50-52$ & 273.2 & 0.63 & 2.17 & 0.27 & 0.14 & 0.09 & 0.00 & 0.04 & & & 0.0 \\
\hline $33 X-2,50-52$ & 278.3 & 0.48 & 1.86 & 0.36 & 0.26 & 0.12 & 0.08 & 0.10 & & & 0.2 \\
\hline $33 X-6,50-52$ & 284.3 & 0.57 & 1.35 & 0.28 & 0.16 & 0.08 & 0.08 & 0.00 & & & 0.2 \\
\hline $34 X-2,48-50$ & 287.88 & 0.64 & 1.56 & 0.40 & 0.50 & 0.08 & 0.43 & 0.00 & 1.40 & 3.0318 & 0.9 \\
\hline $34 X-3,50-52$ & 289.4 & 0.46 & 1.40 & 0.17 & 0.12 & 0.11 & 0.15 & 0.05 & & & 0.3 \\
\hline $34 X-6,50-52$ & 293.86 & 0.44 & 1.85 & 0.22 & 0.19 & 0.17 & 0.37 & 0.05 & 1.27 & 3.0322 & 0.8 \\
\hline $35 X-1,50-52$ & 296 & 0.48 & 1.19 & 0.10 & 0.09 & 0.27 & 0.45 & 0.10 & 0.47 & 3.0346 & 1.0 \\
\hline $35 X-3,50-52$ & 299 & 0.50 & 1.42 & 0.14 & 0.09 & 0.08 & 0.03 & 0.06 & & & 0.1 \\
\hline 311-U1328B- & & & & & & & & & & & \\
\hline $1 \mathrm{H}-2,50-52$ & 2 & 0.53 & 1.58 & 0.16 & 0.22 & 0.18 & 0.20 & 0.08 & & & 0.4 \\
\hline $1 \mathrm{H}-3,50-52$ & 3.5 & 0.67 & 1.74 & 0.15 & 0.38 & 0.14 & 0.19 & 0.09 & & & 0.4 \\
\hline $2 \mathrm{H}-2,50-52$ & 6 & 0.50 & 1.87 & 0.19 & 0.18 & 0.13 & 0.17 & 0.03 & & & 0.4 \\
\hline $5 \mathrm{H}-1,50-52$ & 17 & 0.80 & 1.09 & 0.24 & 0.16 & 0.08 & 0.17 & 0.06 & & & 0.4 \\
\hline $6 X-1,44-46$ & 18.84 & 0.64 & 1.64 & 0.23 & 0.17 & 0.07 & 0.27 & 0.06 & & & 0.5 \\
\hline $8 \mathrm{H}-2,50-52$ & 30 & 0.89 & 1.97 & 0.77 & 0.68 & 0.06 & 0.30 & 0.13 & & & 0.5 \\
\hline $8 \mathrm{H}-4,50-52$ & 33 & 0.81 & 1.72 & 0.26 & 0.51 & 0.11 & 0.09 & 0.00 & & & 0.2 \\
\hline $8 \mathrm{H}-6,49-51$ & 35.49 & 0.76 & 1.38 & 0.67 & 0.79 & 0.06 & 0.28 & 0.11 & & & 0.6 \\
\hline $9 \mathrm{H}-2,50-52$ & 39.47 & 0.77 & 1.27 & 0.29 & 0.16 & 0.12 & 0.18 & 0.05 & & & 0.4 \\
\hline $9 \mathrm{H}-6,50-52$ & 45.31 & 0.54 & 1.44 & 0.24 & 0.18 & 0.10 & 0.43 & 0.00 & 1.43 & 3.0317 & 1.0 \\
\hline $10 \mathrm{H}-2,50-52$ & 49 & 1.05 & 3.66 & 0.22 & 0.10 & 0.10 & 0.04 & 0.00 & & & 0.1 \\
\hline $10 \mathrm{H}-6,50-52$ & 54.97 & 0.72 & 1.52 & 0.17 & 0.13 & 0.15 & 0.00 & 0.00 & & & 0.0 \\
\hline 311-U1328C- & & & & & & & & & & & \\
\hline $1 \mathrm{H}-2,50-52$ & 58.46 & 0.62 & 2.46 & 0.27 & 0.20 & 0.21 & 0.00 & 0.00 & & & 0.0 \\
\hline $1 \mathrm{H}-4,50-52$ & 61.43 & 0.65 & 2.36 & 0.41 & 0.27 & 0.08 & 0.12 & 0.00 & & & 0.3 \\
\hline $1 \mathrm{H}-6,50-52$ & 64.43 & 0.70 & 1.82 & 0.45 & 0.20 & 0.10 & 0.04 & 0.00 & & & 0.1 \\
\hline $2 \mathrm{H}-1,50-52$ & 66.5 & 0.61 & 6.12 & 0.21 & 0.20 & 0.16 & 0.00 & 0.05 & & & 0.0 \\
\hline $3 \mathrm{H}-3,46-48$ & 78.96 & 0.58 & 2.07 & 0.23 & 0.14 & 0.11 & 0.10 & 0.00 & & & 0.2 \\
\hline $3 \mathrm{H}-6,48-50$ & 83.48 & 0.77 & 2.66 & 0.50 & 0.27 & 0.16 & 0.00 & 0.00 & & & 0.0 \\
\hline $4 X-1,50-52$ & 85.5 & 0.72 & 1.75 & 0.35 & 0.16 & 0.11 & 0.00 & 0.00 & & & 0.0 \\
\hline $4 X-3,41-43$ & 88.41 & 0.54 & 1.92 & 0.23 & 0.10 & 0.08 & 0.07 & 0.00 & & & 0.2 \\
\hline $6 \mathrm{H}-2,50-52$ & 96 & 0.54 & 1.49 & 0.35 & 0.20 & 0.13 & 0.00 & 0.00 & & & 0.0 \\
\hline $6 \mathrm{H}-4,50-52$ & 98.4 & 0.44 & 1.03 & 0.10 & 0.11 & 0.11 & 0.06 & 0.00 & & & 0.1 \\
\hline $6 \mathrm{H}-6,50-52$ & 99.5 & 0.32 & 1.31 & 0.17 & 0.17 & 0.06 & 0.00 & 0.00 & & & 0.0 \\
\hline $7 X-1,50-52$ & 104 & 0.59 & 1.38 & 0.30 & 0.30 & 0.10 & 0.85 & 0.19 & 0.57 & 3.0343 & 1.9 \\
\hline $7 X-2,50-52$ & 105.5 & 0.53 & 1.38 & 0.17 & 0.14 & 0.09 & 0.50 & 0.04 & 0.77 & 3.0337 & 1.1 \\
\hline
\end{tabular}


Table T1 (continued).

\begin{tabular}{|c|c|c|c|c|c|c|c|c|c|c|c|}
\hline \multirow{2}{*}{$\begin{array}{l}\text { Core, section, } \\
\text { interval }(\mathrm{cm})\end{array}$} & \multirow{2}{*}{$\begin{array}{l}\text { Depth } \\
\text { (mbsf) }\end{array}$} & \multirow{2}{*}{$\begin{array}{l}\text { I, quartz } \\
(4.26 \AA)\end{array}$} & \multirow{2}{*}{$\begin{array}{c}\text { A, feldspars } \\
(3.18-3.24 \AA)\end{array}$} & \multirow{2}{*}{$\begin{array}{c}\text { I, chlorite } \\
(3.54 \AA)\end{array}$} & \multirow{2}{*}{$\begin{array}{l}\text { I, illite } \\
(10 \AA)\end{array}$} & \multirow{2}{*}{$\begin{array}{c}\text { l, pyrite } \\
(2.706 \AA)\end{array}$} & \multirow{2}{*}{$\begin{array}{l}\text { I, calcite } \\
\text { d(104) }\end{array}$} & \multirow{2}{*}{$\begin{array}{l}\text { I, dolomite } \\
\mathrm{d}(104)\end{array}$} & $\mathrm{MgCO}_{3}$ & Calci & \\
\hline & & & & & & & & & $(\mathrm{mol} \%)$ & $\mathrm{d}(104)(\AA)$ & (wt\%) \\
\hline $7 X-4,50-52$ & 108.5 & 0.77 & 1.99 & 0.22 & 0.09 & 0.19 & 0.14 & 0.00 & & & 0.3 \\
\hline $7 X-5,52-54$ & 109.92 & 0.77 & 1.50 & 0.34 & 0.12 & 0.10 & 0.08 & 0.04 & & & 0.2 \\
\hline $8 X-2,50-52$ & 112.3 & 1.18 & 4.11 & 0.33 & 0.27 & 0.10 & 0.11 & 0.00 & & & 0.2 \\
\hline $8 X-4,44-46$ & 114.92 & 0.65 & 1.94 & 0.28 & 0.13 & 0.08 & 0.00 & 0.00 & & & 0.0 \\
\hline $8 X-6,50-52$ & 117.98 & 0.69 & 1.55 & 0.29 & 0.14 & 0.13 & 0.07 & 0.00 & & & 0.1 \\
\hline $9 X-2,52-54$ & 121.92 & 0.68 & 4.61 & 0.23 & 0.11 & 0.06 & 0.06 & 0.00 & & & 0.1 \\
\hline $9 X-4,46-48$ & 124.36 & 1.04 & 2.95 & 0.35 & 0.15 & 0.07 & 0.22 & 0.00 & & & 0.5 \\
\hline $9 X-6,37-39$ & 127.14 & 0.69 & 1.60 & 0.29 & 0.13 & 0.11 & 0.00 & 0.00 & & & 0.0 \\
\hline $11 X-2,50-52$ & 141.3 & 0.56 & 1.62 & 0.24 & 0.21 & 0.13 & 0.04 & 0.00 & & & 0.1 \\
\hline $11 X-4,50-52$ & 144.3 & 0.47 & 1.64 & 0.27 & 0.28 & 0.14 & 0.28 & 0.05 & & & 0.6 \\
\hline $12 X-2,50-52$ & 150.9 & 0.75 & 2.56 & 0.21 & 0.22 & 0.13 & 0.23 & 0.00 & & & 0.5 \\
\hline $12 X-4,50-52$ & 153.9 & 0.75 & 2.53 & 0.32 & 0.29 & 0.14 & 0.16 & 0.07 & & & 0.4 \\
\hline $12 X-6,51-53$ & 156.41 & 0.51 & 1.81 & 0.28 & 0.19 & 0.11 & 0.15 & 0.00 & & & 0.4 \\
\hline $13 X-2,148-150$ & 161.55 & 0.68 & 1.57 & 0.34 & 0.37 & 0.07 & 0.43 & 0.10 & 1.03 & 3.0329 & 1.0 \\
\hline $13 X-4,50-52$ & 163.57 & 0.69 & 1.63 & 0.36 & 0.36 & 0.20 & 0.29 & 0.13 & & & 0.6 \\
\hline $14 X-2,50-52$ & 170.03 & 0.67 & 2.31 & 0.52 & 0.35 & 0.16 & 0.00 & 0.00 & & & 0.0 \\
\hline $15 X-2,45-47$ & 179.85 & 0.59 & 2.59 & 0.27 & 0.18 & 0.17 & 0.00 & 0.00 & & & 0.0 \\
\hline $15 X-4,50-52$ & 182.87 & 0.59 & 2.31 & 0.32 & 0.20 & 0.15 & 0.00 & 0.06 & & & 0.0 \\
\hline $16 X-2,50-52$ & 189.5 & 0.46 & 1.05 & 0.09 & 0.10 & 0.10 & 0.65 & 0.04 & 0.50 & 3.0345 & 1.5 \\
\hline $17 X-2,50-52$ & 199.1 & 0.78 & 1.68 & 0.25 & 0.26 & 0.11 & 0.72 & 0.00 & 0.90 & 3.0333 & 1.6 \\
\hline $17 X-4,50-52$ & 202.1 & 0.42 & 1.30 & 0.07 & 0.10 & 0.11 & 0.67 & 0.05 & 0.20 & 3.0354 & 1.4 \\
\hline $17 X-6,50-52$ & 204.6 & 0.79 & 1.48 & 0.20 & 0.23 & 0.12 & 0.76 & 0.05 & 1.03 & 3.0329 & 1.7 \\
\hline $18 X-3,50-52$ & 209.5 & 0.50 & 1.40 & 0.17 & 0.19 & 0.18 & 1.04 & 0.07 & 0.77 & 3.0337 & 2.0 \\
\hline $19 X-2,50-52$ & 217.53 & 0.50 & 1.97 & 0.16 & 0.14 & 0.18 & 0.25 & 0.00 & & & 0.5 \\
\hline $19 X-4,50-52$ & 220.53 & 0.54 & 1.00 & 0.38 & 0.24 & 0.09 & 0.52 & 0.05 & 0.83 & 3.0335 & 1.1 \\
\hline $20 X-2,50-52$ & 228 & 0.57 & 1.84 & 0.22 & 0.14 & 0.15 & 0.60 & 0.00 & 0.73 & 3.0338 & 1.3 \\
\hline $21 X-2,50-52$ & 237.38 & 0.54 & 1.95 & 0.17 & 0.13 & 0.26 & 0.14 & 0.05 & & & 0.3 \\
\hline $21 X-6,50-52$ & 243.27 & 0.54 & 1.89 & 0.21 & 0.14 & 0.13 & 0.57 & 0.00 & 1.23 & 3.0323 & 1.2 \\
\hline $22 X-2,47-49$ & 247.17 & 0.88 & 2.15 & 0.23 & 0.08 & 0.15 & 0.00 & 0.06 & & & 0.0 \\
\hline $22 X-6,50-52$ & 252.31 & 0.60 & 2.10 & 0.24 & 0.18 & 0.26 & 0.00 & 0.00 & & & 0.0 \\
\hline $23 X-2,50-52$ & 256.8 & 0.64 & 2.01 & 0.18 & 0.09 & 0.16 & 0.04 & 0.08 & & & 0.1 \\
\hline $23 X-4,50-52$ & 259.8 & 0.54 & 1.75 & 0.20 & 0.14 & 0.22 & 0.38 & 0.00 & 1.30 & 3.0321 & 0.8 \\
\hline $24 X-2,53-55$ & 266.39 & 0.47 & 1.91 & 0.21 & 0.10 & 0.11 & 0.26 & 0.05 & 0.83 & 3.0335 & 0.6 \\
\hline $24 X-4,48-50$ & 269.22 & 0.62 & 1.79 & 0.24 & 0.13 & 0.14 & 0.06 & 0.00 & & & 0.1 \\
\hline $24 X-6,50-52$ & 271 & 0.49 & 1.80 & 0.26 & 0.20 & 0.21 & 0.00 & 0.00 & & & 0.0 \\
\hline $25 X-2,50-52$ & 276.1 & 0.56 & 2.39 & 0.37 & 0.16 & 0.15 & 0.05 & 0.00 & & & 0.1 \\
\hline $25 X-4,52-54$ & 279.12 & 0.61 & 1.73 & 0.29 & 0.17 & 0.16 & 0.00 & 0.00 & & & 0.0 \\
\hline $25 X-6,50-52$ & 281.6 & 1.12 & 1.62 & 0.23 & 0.18 & 0.23 & 0.07 & 0.00 & & & 0.2 \\
\hline $26 X-2,50-52$ & 285.7 & 0.63 & 1.83 & 0.21 & 0.16 & 0.16 & 0.07 & 0.00 & & & 0.2 \\
\hline $26 \times-4,50-52$ & 288.7 & 0.54 & 1.49 & 0.22 & 0.22 & 0.14 & 0.14 & 0.07 & & & 0.3 \\
\hline $26 X-6,49-51$ & 291.69 & 0.72 & 2.77 & 0.19 & 0.11 & 0.15 & 0.07 & 0.00 & & & 0.1 \\
\hline $27 X-2,50-52$ & 295.3 & 0.61 & 1.42 & 0.22 & 0.14 & 0.08 & 0.00 & 0.00 & & & 0.0 \\
\hline $27 X-6,50-52$ & 300.61 & 0.60 & 1.86 & 0.15 & 0.12 & 0.13 & 0.36 & 0.07 & 1.10 & 3.0327 & 0.8 \\
\hline 311-U1328D- & & & & & & & & & & & \\
\hline $1 X-2,56-58$ & 2.06 & 0.80 & 1.42 & 0.21 & 0.19 & 0.19 & 0.23 & 0.00 & & & 0.5 \\
\hline $2 X-2,32-34$ & 5.82 & 0.76 & 1.06 & 0.18 & 0.26 & 0.11 & 0.18 & 0.00 & & & 0.4 \\
\hline 311-U1328E- & & & & & & & & & & & \\
\hline $2 X-1,50-52$ & 7 & 0.58 & 1.43 & 0.19 & 0.28 & 0.13 & 0.21 & 0.00 & & & 0.5 \\
\hline $8 X-1,28-30$ & 26.98 & 0.74 & 1.83 & 0.62 & 0.68 & 0.12 & 0.25 & 0.00 & & & 0.6 \\
\hline $8 X-1,74-76$ & 27.44 & 0.84 & 2.54 & 0.50 & 0.52 & 0.11 & 0.18 & 0.00 & & & 0.4 \\
\hline $8 X-1,95-97$ & 27.65 & 0.94 & 0.95 & 0.65 & 0.57 & 0.10 & 0.29 & 0.00 & & & 0.6 \\
\hline 311-U1329B- & & & & & & & & & & & \\
\hline $1 \mathrm{H}-2,48-50$ & 1.98 & 0.65 & 2.03 & 0.36 & 0.24 & 0.15 & 0.50 & 0.08 & 0.43 & 3.0347 & 1.1 \\
\hline $1 \mathrm{H}-4,42-44$ & 5.02 & 0.74 & 1.41 & 0.33 & 0.35 & 0.14 & 0.52 & 0.18 & 0.80 & 3.0336 & 1.1 \\
\hline $1 \mathrm{H}-6,50-52$ & 8 & 0.61 & 1.41 & 0.19 & 0.20 & 0.09 & 0.35 & 0.04 & 1.23 & 3.0323 & 0.7 \\
\hline 311-U1329C- & & & & & & & & & & & \\
\hline $1 \mathrm{H}-2,50-52$ & 2 & 0.65 & 2.48 & 0.29 & 0.14 & 0.12 & 0.12 & 0.00 & & & 0.3 \\
\hline $1 \mathrm{H}-4,50-52$ & 5 & 0.77 & 1.71 & 0.14 & 0.11 & 0.11 & 0.27 & 0.04 & & & 0.7 \\
\hline $2 \mathrm{H}-2,50-52$ & 10.1 & 0.70 & 1.69 & 0.15 & 0.33 & 0.20 & 0.79 & 0.20 & 1.17 & 3.0325 & 1.7 \\
\hline $2 \mathrm{H}-4,50-52$ & 13.1 & 0.79 & 2.34 & 0.26 & 0.12 & 0.09 & 0.23 & 0.04 & & & 0.5 \\
\hline $2 \mathrm{H}-6,50-52$ & 16.1 & 0.61 & 2.00 & 0.49 & 0.56 & 0.17 & 0.26 & 0.00 & & & 0.6 \\
\hline $3 \mathrm{H}-2,43-45$ & 19.53 & 0.74 & 1.48 & 0.24 & 0.15 & 0.12 & 0.77 & 0.27 & 0.87 & 3.0334 & 1.7 \\
\hline $3 \mathrm{H}-3,43-45$ & 21.03 & 0.51 & 1.90 & 0.20 & 0.17 & 0.15 & 1.32 & 0.19 & 0.27 & 3.0352 & 3.0 \\
\hline $3 \mathrm{H}-6,49-51$ & 25.59 & 0.80 & 1.94 & 0.21 & 0.11 & 0.14 & 0.14 & 0.00 & & & 0.3 \\
\hline $4 \mathrm{H}-2,50-52$ & 29.1 & 0.55 & 1.77 & 0.26 & 0.25 & 0.15 & 0.54 & 0.07 & 1.23 & 3.0323 & 1.2 \\
\hline $4 \mathrm{H}-4,50-52$ & 32.1 & 0.53 & 1.58 & 0.39 & 0.34 & 0.16 & 0.40 & 0.00 & 1.70 & 3.0309 & 0.9 \\
\hline $4 \mathrm{H}-6,53-55$ & 35.13 & 0.53 & 1.99 & 0.25 & 0.16 & 0.16 & 0.48 & 0.00 & 1.23 & 3.0323 & 1.0 \\
\hline
\end{tabular}


Table T1 (continued).

\begin{tabular}{|c|c|c|c|c|c|c|c|c|c|c|c|}
\hline \multirow{2}{*}{$\begin{array}{l}\text { Core, section, } \\
\text { interval }(\mathrm{cm})\end{array}$} & \multirow{2}{*}{$\begin{array}{l}\text { Depth } \\
\text { (mbsf) }\end{array}$} & \multirow{2}{*}{$\begin{array}{l}\text { I, quartz } \\
(4.26 \AA)\end{array}$} & \multirow{2}{*}{$\begin{array}{c}\text { A, feldspars } \\
(3.18-3.24 \AA)\end{array}$} & \multirow{2}{*}{$\begin{array}{c}\text { I, chlorite } \\
(3.54 \AA)\end{array}$} & \multirow{2}{*}{$\begin{array}{l}\text { I, illite } \\
(10 \AA)\end{array}$} & \multirow{2}{*}{$\begin{array}{c}\text { l, pyrite } \\
(2.706 \AA)\end{array}$} & \multirow{2}{*}{$\begin{array}{l}\text { I, calcite } \\
\mathrm{d}(104)\end{array}$} & \multirow{2}{*}{$\begin{array}{l}\text { I, dolomite } \\
\text { d(104) }\end{array}$} & \multirow{2}{*}{$\begin{array}{l}\mathrm{MgCO}_{3} \\
(\mathrm{~mol} \%)\end{array}$} & \multicolumn{2}{|c|}{ Calcite } \\
\hline & & & & & & & & & & $\mathrm{d}(104)(\AA)$ & (wt\%) \\
\hline $5 \mathrm{H}-2,50-52$ & 38.6 & 0.59 & 1.39 & 0.46 & 0.44 & 0.11 & 0.26 & 0.17 & & & 0.6 \\
\hline $5 \mathrm{H}-3,127-129$ & 40.87 & 0.44 & 1.43 & 0.11 & 0.13 & 0.13 & 0.93 & 0.03 & 6.57 & 3.0163 & 2.3 \\
\hline $5 \mathrm{H}-4,50-52$ & 41.6 & 0.80 & 2.08 & 0.18 & 0.23 & 0.13 & 0.00 & 0.02 & & & 0.0 \\
\hline $5 \mathrm{H}-6,50-52$ & 44.6 & 0.39 & 1.40 & 0.20 & 0.20 & 0.31 & 0.34 & 0.07 & 1.67 & 3.0310 & 0.8 \\
\hline $6 \mathrm{H}-2,50-52$ & 48.1 & 0.68 & 2.66 & 0.13 & 0.11 & 0.19 & 0.24 & 0.03 & & & 0.6 \\
\hline $6 \mathrm{H}-4,50-52$ & 51.1 & 0.48 & 1.91 & 0.31 & 0.15 & 0.15 & 0.06 & 0.00 & & & 0.1 \\
\hline $6 \mathrm{H}-6,50-52$ & 54.1 & 0.61 & 2.46 & 0.31 & 0.16 & 0.20 & 0.06 & 0.00 & & & 0.1 \\
\hline $8 \mathrm{H}-2,46-48$ & 59.56 & 0.57 & 2.41 & 0.15 & 0.16 & 0.20 & 0.07 & 0.04 & & & 0.1 \\
\hline $8 \mathrm{H}-4,50-52$ & 62.6 & 0.52 & 1.96 & 0.36 & 0.29 & 0.15 & 0.64 & 0.00 & 0.83 & 3.0335 & 1.3 \\
\hline $8 \mathrm{H}-6,50-52$ & 65.6 & 0.64 & 2.60 & 0.27 & 0.11 & 0.16 & 0.34 & 0.00 & & & 0.7 \\
\hline $9 \mathrm{H}-2,50-52$ & 69.1 & 0.46 & 2.21 & 0.43 & 0.46 & 0.23 & 0.90 & 0.00 & 0.60 & 3.0342 & 1.9 \\
\hline $9 \mathrm{H}-4,46-48$ & 72.06 & 0.55 & 1.41 & 0.38 & 0.31 & 0.13 & 1.30 & 0.00 & 0.80 & 3.0336 & 2.7 \\
\hline $9 \mathrm{H}-6,50-52$ & 75.1 & 0.59 & 1.72 & 0.32 & 0.30 & 0.24 & 0.56 & 0.10 & 1.03 & 3.0329 & 1.1 \\
\hline $10 \mathrm{H}-2,50-52$ & 78.6 & 0.64 & 1.77 & 0.19 & 0.19 & 0.14 & 0.56 & 0.03 & 1.23 & 3.0323 & 1.1 \\
\hline $10 \mathrm{H}-4,50-52$ & 81.6 & 0.74 & 3.19 & 0.32 & 0.23 & 0.18 & 0.36 & 0.03 & 0.87 & 3.0334 & 0.8 \\
\hline $11 \mathrm{H}-2,50-52$ & 88.1 & 0.51 & 1.93 & 0.13 & 0.13 & 0.14 & 0.58 & 0.00 & 0.00 & 3.0360 & 1.3 \\
\hline $11 \mathrm{H}-4,46-48$ & 91.06 & 0.45 & 1.92 & 0.17 & 0.18 & 0.14 & 0.16 & 0.05 & & & 0.3 \\
\hline $11 \mathrm{H}-6,50-52$ & 94.1 & 0.54 & 1.97 & 0.33 & 0.39 & 0.13 & 0.16 & 0.06 & & & 0.4 \\
\hline $12 \mathrm{H}-2,50-52$ & 97.58 & 0.50 & 1.42 & 0.43 & 0.48 & 0.12 & 0.34 & 0.20 & 1.93 & 3.0302 & 0.8 \\
\hline $12 \mathrm{H}-4,50-52$ & 100.58 & 0.64 & 1.85 & 0.27 & 0.20 & 0.10 & 0.35 & 0.00 & 0.90 & 3.0333 & 0.7 \\
\hline $12 \mathrm{H}-6,50-52$ & 103.58 & 0.61 & 1.84 & 0.29 & 0.14 & 0.14 & 0.06 & 0.05 & & & 0.1 \\
\hline $13 \mathrm{H}-2,50-52$ & 107.1 & 0.52 & 1.66 & 0.20 & 0.11 & 0.14 & 0.27 & 0.06 & & & 0.6 \\
\hline $13 \mathrm{H}-4,50-52$ & 110.1 & 0.58 & 2.28 & 0.27 & 0.16 & 0.21 & 0.10 & 0.00 & & & 0.2 \\
\hline $13 \mathrm{H}-6,50-52$ & 113.1 & 0.52 & 1.47 & 0.34 & 0.23 & 0.14 & 0.27 & 0.06 & & & 0.6 \\
\hline $15 \mathrm{H}-2,50-52$ & 118.6 & 0.48 & 1.84 & 0.15 & 0.14 & 0.06 & 0.04 & 0.00 & & & 0.1 \\
\hline $15 \mathrm{H}-4,50-52$ & 121.6 & 0.63 & 1.70 & 0.13 & 0.12 & 0.15 & 0.00 & 0.00 & & & 0.0 \\
\hline $15 \mathrm{H}-6,50-52$ & 124.6 & 0.61 & 1.71 & 0.15 & 0.11 & 0.08 & 0.03 & 0.00 & & & 0.1 \\
\hline $16 \mathrm{H}-2,61-63$ & 128.21 & 0.68 & 1.94 & 0.26 & 0.15 & 0.06 & 0.43 & 0.00 & 0.63 & 3.0341 & 1.0 \\
\hline $16 \mathrm{H}-4,50-52$ & 131.1 & 0.62 & 2.78 & 0.34 & 0.29 & 0.16 & 0.00 & 0.05 & & & 0.0 \\
\hline $16 \mathrm{H}-6,50-52$ & 134.1 & 0.51 & 2.08 & 0.10 & 0.10 & 0.12 & 0.00 & 0.03 & & & 0.0 \\
\hline $17 \mathrm{H}-2,50-52$ & 137.6 & 0.55 & 1.17 & 0.01 & 0.10 & 0.11 & 0.00 & 0.00 & & & 0.0 \\
\hline $18 X-2,50-52$ & 142.2 & 0.59 & 1.53 & 0.17 & 0.13 & 0.14 & 0.04 & 0.00 & & & 0.1 \\
\hline $18 X-4,50-52$ & 145.2 & 0.50 & 1.51 & 0.12 & 0.08 & 0.13 & 0.08 & 0.00 & & & 0.2 \\
\hline $19 X-4,50-52$ & 154.9 & 0.69 & 1.38 & 0.15 & 0.11 & 0.09 & 0.09 & 0.00 & & & 0.2 \\
\hline $19 X-6,50-52$ & 157.4 & 0.57 & 1.25 & 0.18 & 0.06 & 0.10 & 0.00 & 0.00 & & & 0.0 \\
\hline $20 X-4,50-52$ & 164.6 & 0.50 & 1.23 & 0.06 & 0.09 & 0.15 & 0.00 & 0.00 & & & 0.0 \\
\hline $20 \times-4,80-82$ & 164.9 & 0.51 & 1.31 & 0.07 & 0.09 & 0.16 & 0.02 & 0.06 & & & 0.1 \\
\hline $21 X-2,50-52$ & 171.3 & 0.53 & 1.53 & 0.12 & 0.06 & 0.13 & 0.07 & 0.00 & & & 0.2 \\
\hline $21 X-4,50-52$ & 174.3 & 0.51 & 1.14 & 0.05 & 0.09 & 0.12 & 0.04 & 0.00 & & & 0.1 \\
\hline $22 X-2,50-52$ & 180.9 & 0.51 & 2.62 & 0.20 & 0.17 & 0.17 & 0.04 & 0.03 & & & 0.1 \\
\hline \multicolumn{12}{|l|}{ 311-U1329E- } \\
\hline $1 \mathrm{H}-2,50-52$ & 2 & 0.61 & 1.63 & 0.20 & 0.09 & 0.22 & 0.06 & 0.06 & & & 0.1 \\
\hline $2 \mathrm{H}-2,50-52$ & 7 & 0.64 & 1.77 & 0.25 & 0.18 & 0.18 & 0.28 & 0.05 & & & 0.6 \\
\hline $2 \mathrm{H}-4,50-52$ & 10 & 0.57 & 1.55 & 0.22 & 0.22 & 0.20 & 0.29 & 0.05 & & & 0.6 \\
\hline $2 \mathrm{H}-6,50-52$ & 13 & 0.86 & 2.44 & 0.30 & 0.36 & 0.11 & 0.71 & 0.11 & 0.27 & 3.0352 & 1.5 \\
\hline $3 \mathrm{H}-2,50-52$ & 16.5 & 0.72 & 1.84 & 0.76 & 0.75 & 0.15 & 0.37 & 0.23 & & & 0.7 \\
\hline $3 \mathrm{H}-4,50-52$ & 19.5 & 0.56 & 1.64 & 0.14 & 0.12 & 0.12 & 0.57 & 0.09 & 0.27 & 3.0352 & 1.1 \\
\hline $3 \mathrm{H}-6,50-52$ & 22.5 & 0.63 & 1.49 & 0.13 & 0.10 & 0.15 & 0.49 & 0.00 & 0.83 & 3.0335 & 1.0 \\
\hline $4 \mathrm{H}-2,50-52$ & 26 & 0.48 & 1.83 & 0.24 & 0.20 & 0.13 & 0.04 & 0.04 & & & 0.1 \\
\hline $4 \mathrm{H}-4,50-52$ & 29 & 0.74 & 1.75 & 0.51 & 0.42 & 0.25 & 0.55 & 0.07 & 0.83 & 3.0335 & 1.0 \\
\hline $4 \mathrm{H}-6,50-52$ & 32 & 0.63 & 1.57 & 0.17 & 0.20 & 0.16 & 0.31 & 0.18 & 1.40 & 3.0318 & 0.7 \\
\hline
\end{tabular}

Note: XRD peak-intensity $(I)$ or area $(A)$ relative to I or $A$ of corundum standard $(2.085 \AA)$. 